\title{
Quadrupole Sensitive Pulse for Signal Filtering
}

\author{
Nimerovsky Evgeny ${ }^{l}$, Alexej Jerschow ${ }^{1} *$ \\ ${ }^{1}$ Department of Chemistry, New York University, New York, NY 10003, USA \\ *corresponding author:email: alexej.jerschow@nyu.edu; Phone: 1-212-998-8451 \\ Address: Silver Center for Arts and Science, 100 Washington Square East, New York University, New York, NY 10003, USA
}

\begin{abstract}
A longstanding problem in quadrupolar NMR of semi-solids is the selection of signals originating from ordered nuclei, i.e. those that experience a non-vanishing quadrupolar coupling. Established techniques, such as for example multiple-quantum filters are not adequate in situations when the radio frequency power is on the order of the quadrupolar coupling or the quadrupolar relaxation rates, such as may be the case on an MRI scanner, or in ex situ applications. In this manuscript we show a new method for the selective excitation of ordered spin-3/2 nuclei, which produces the desired results when the radio frequency power is approximately equal or smaller than quadrupolar frequency. Using a combination of simulations and experiments with ${ }^{23} \mathrm{Na}$ in $\mathrm{NaCl}$ solution, $\mathrm{Pf} 1$-solutions, and bovine patellar cartilage samples we further show how the value of the quadrupolar frequency and global features of a quadrupolar coupling distribution can be extracted from these experiments.
\end{abstract}

\section{Introduction}

Sodium ions play important roles in human physiology. In the body, sodium ions can either exist in their free form, or restricted in their motion. Typically, the extracellular compartment contains freely moving sodium ions, with a few exceptions, such as for example cartilage tissue, where sodium ions are associated with macromolecular constructs, and motion is restricted via binding to negatively charged entitites. ${ }^{1,2}$ Anisotropic motion, or motion with partial averaging leads to the appearance of a residual quadrupolar coupling. ${ }^{3,4}$ Biexponentional relaxation is observed when sodium ions are tumbling relatively slowly, such as in the crowded intra-cellular environment ${ }^{5}$. Although the concentration of the extracellular sodium is about 10 times larger than the one of intra-cellular sodium, monitoring the latter and sodium in cartilage tissue is of greater interest for indicating physiological changes ${ }^{2,6}$. Several methods exist for diagnosing either restricted or slow motion, which include the multiple quantum filtered sequence $^{7-18}$, sequences based on mixed selective and non-selective pulse sequences ${ }^{19,20}$ and a quadrupolar jump-and-return sequence ${ }^{21}$. The multiple-quantum filtered sequences, although very powerful, require strong pulses and a relatively long phase cycle for these to be useful. In cases where the quadrupolar frequency $v_{Q}$ is not significantly smaller than the Rabi frequency $v_{r f}$, large losses and artifacts are observed, which prevent these methods from being used reliably in vivo ${ }^{7,22}$.

In this article, we describe a new method, called Quadrupole Sensitive Pulse (QSP), for the selective excitation of ordered quadrupolar spins only (i.e. those that display residual quadrupolar couplings) when the radio frequency fields (rf-field, $v_{r f}$ ) of the pulses are approximately equal or smaller than a given quadrupolar frequency. One feature of QSP is that it is a central transition $(C T)$ measurement and therefore it can also be used as either a preparation module or an excitation module in other sequences, 
such as for example in spin echo and inversion recovery sequences (the $90^{\circ}$ pulse can be replaced with QSP for excitation of ordered spins). The second feature of this method is the dependence of the intensity of the $C T$ on the quadrupolar frequency - $C T\left(v_{Q}\right)$. By performing this experiment as a function of the total duration of the sequence, we are able to determine the value of the quadrupolar frequency or global features of a quadrupolar coupling distribution.

\section{Theory}

Experiments in which the internal interactions are much smaller or much larger than $v_{r f}$ are well known and most of them have analytical solutions for the action of the rf-field. We consider here in particular the quadrupolar coupling, which arises from the interaction between the quadrupole moment of the nucleus $(S>1 / 2)$ and a gradient of the electric field ${ }^{23}$. The value of the quadrupolar frequency $\left(v_{Q}(\right.$ in $\mathrm{Hz})=$ $\left.\frac{3 e^{2} q Q}{2 S(2 S-1)}\right)$ quantifies the electric charge anisotropy surrounding the nucleus. The quadrupole Hamiltonian in the high field approximation is also anisotropic and it depends on orientation with respect to the Zeeman field. The first order quadrupole Hamiltonian in a static solid ${ }^{24}$ can be written as:

$$
H_{Q}=\frac{v_{Q}}{2}\left[\frac{3 \cos ^{2} \beta-1}{2}\right]\left(S_{z}^{2}-\frac{1}{3} S(S+1)\right),
$$

in units of $\mathrm{Hz}$, where we have assumed for simplicity $\eta_{Q}=0$. Here $\beta$ is the angle between the magnetic field and the principal axis frame of the quadrupole tensor.

Investigating the sample with an orientational parallel to the magnetic field, Eq. (1) can be simplified to

$$
H_{Q}=\frac{v_{Q}}{2}\left(S_{z}^{2}-\frac{1}{3} S(S+1)\right),
$$

where we have taken $\beta=0$.

The observed signal is the trace of the multiplication of the appropriate detection operator and the density matrix,

$$
S\left(t ; v_{Q}, \beta=0\right)=\operatorname{Tr}\left\{A^{\dagger} \rho\left(t, v_{Q}\right)\right\}
$$

where $A=S^{-}$, and it can in many cases be reduced to just $S_{y}$ (e.g. in the absence of offset effects) ; $\rho\left(t, v_{Q}\right)$ is the density matrix.

In the case of a random distribution of principal axis frames, the signal as given by Eq. (3) is averaged over all orientations,

$$
S\left(t ; v_{Q}\right) \propto \int d \alpha d(\cos \beta) S\left(t ; v_{Q}, \beta\right)
$$

In biological tissues, the dependence of the quadrupolar coupling distribution is more complicated because of potentially anisotropic orientational distributions ${ }^{25}$. To encompass these situations, we write the more general form using averaging over a quadrupolar frequency distribution in addition to orientational averaging ${ }^{9,26}$, 


$$
S\left(\mathrm{t} ; v_{Q}^{l o c}, \Delta v_{Q}^{l o c}\right) \propto \int d v_{Q} W_{Q}\left(v_{Q} ; v_{Q}^{l o c}, \Delta v_{Q}^{l o c}\right) \int d \alpha d(\cos \beta) S\left(t ; v_{Q}, \beta\right)
$$

where $W_{Q}\left(v_{Q} ; v_{Q}^{l o c}, \Delta v_{Q}^{l o c}\right)$ is the weight factor. This weight factor is often described well by a Gaussian distribution, ${ }^{9}$ and occasionally by a parabolic function ${ }^{12}$.

The spectra of sodium with parallel orientation, in a powder or in biological tissues are well known ${ }^{3}$. For our purpose, we will consider the behavior of central transitions only.

The density matrix can be written as:

$$
\rho\left(t, v_{Q}\right)=U\left(t, v_{Q}\right) \rho(0) U^{-1}\left(t, v_{Q}\right)
$$

where

$$
U\left(t, v_{Q}\right)=\left\{\begin{array}{c}
\exp \left\{-i t\left(H_{r f}+H_{Q}\right)\right\} \quad v_{r f} \neq 0 \\
\exp \left\{-i t H_{Q}\right\} \quad v_{r f}=0
\end{array} .\right.
$$

If $v_{r f} \gg v_{Q}$ the quadrupole interaction can be neglected during the pulse and the value of the central transition depends on the flip angle of the pulse (Fig. 1, black peaks; the flip angle is $\pi / 4$ ). If $v_{r f} \ll v_{Q}$, the pulse influences the central transition only and therefore the quadrupole interaction can be neglected during the pulse also (Fig. 1, blue peaks). If $v_{r f} \sim v_{Q}$ the behavior of the central transition is more complicated and depends on the quadrupolar frequency (Fig. 1, red peak).

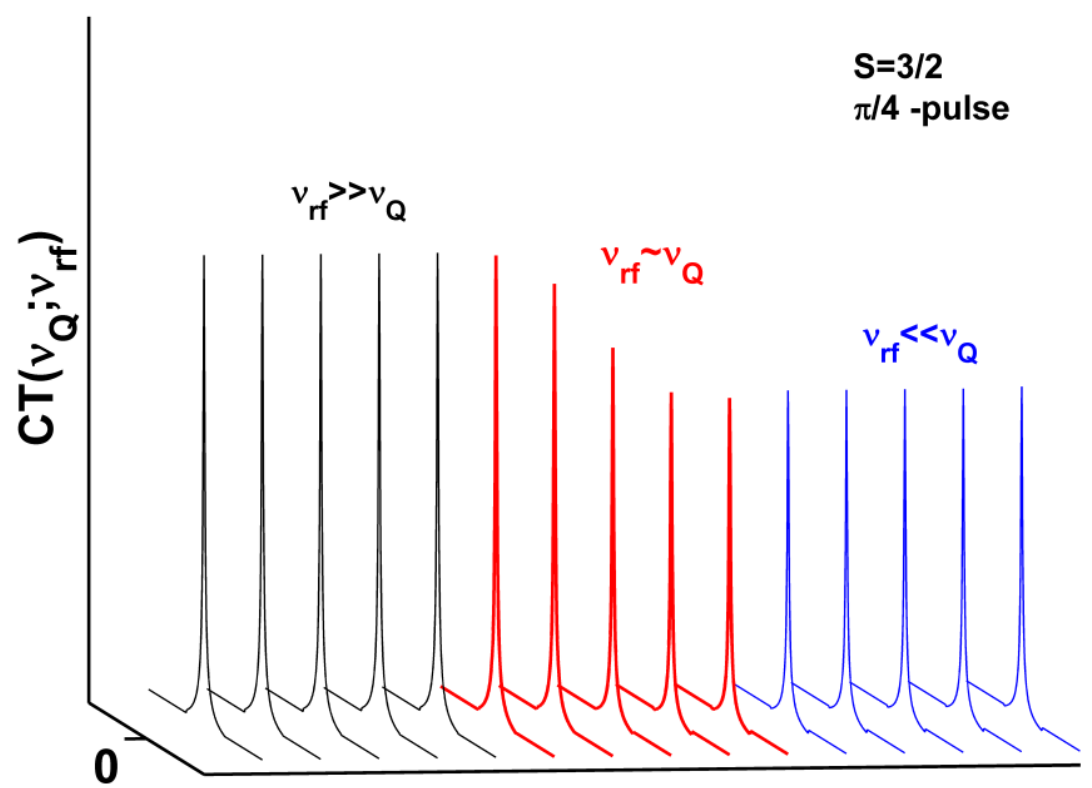

Fig. 1 Dependence of the intensity of the central transition $(C T)$ on the ratio of $v_{r f} / v_{Q}: v_{r f} \gg v_{Q}$ (black peaks) $v_{r f}=$ $100 \mathrm{kHz} ; v_{Q}=1,2,3,4,5(\mathrm{kHz}) ; v_{r f} \sim v_{Q}$ (red peaks), $v_{r f}=1 \mathrm{kHz} ; v_{Q}=0.5,2,4,6,8(\mathrm{kHz}) ; v_{r f} \ll v_{Q}$ (blue peaks), $v_{r f}=1.1 \mathrm{kHz} ; v_{Q}=110,120,130,140,150(\mathrm{kHz})$. The flip angle of the pulse is $\pi / 4$.

Theoretical solutions in the regime where $v_{r f} \sim v_{Q}$ are difficult to derive, and when they exist, they are very bulky and do not provide much insight into the physics of the experiments. On the other hand, the 
quadrupolar couplings in biological tissues are frequently in the range of 500-3000 $\mathrm{Hz}^{4}$ and therefore, the regime $v_{r f} \sim v_{Q}$ can be used for the selective excitation and observation of ordered sodium. The required conditions for such a pulse sequence are:

1. Excitation of spins only when they have a certain minimum quadrupolar coupling;

2. Small dependence of performance on the inhomogeneity of the external field;

3. Small dependence of performance on the inhomogeneity of the rf-field.

The first condition can be achieved using two adjacent pulses with the same duration and alternating phases $\left(0^{\circ}\right.$ and $\left.180^{\circ}\right)$. The second and third conditions can be achieved using a $\pi$-pulse and phase cycling of the $\pi$-pulse. This approach is similar to the quadrupolar jump-and-return sequence ${ }^{21}$, except that now we are focusing on a case where the pulses cannot be used at powers that significantly exceed the quadrupolar couplings (such as for sodium imaging in vivo, for example).

The follow sequence, called Quadrupole Sensitive Pulse (QSP), satisfies all these conditions (Fig. 2): two pairs of adjacent pulses with alternating phases, and a $\pi$-pulse between them with a two-step phase cycle $\left(0^{\circ}, 180^{\circ}\right)$. The durations of the pulses (except the $\pi$-pulse) are chosen as

$$
t_{p, \kappa}=\frac{1}{4 v_{r f}} \frac{1}{\kappa} ; \quad \kappa \geq 1.25 .
$$

The condition in Eq. (8) was found experimentally. All experimental points under $\kappa=1$ (in these cases the flip angles of the four pulses surrounding the $180^{\circ}$ pulse have a flip angle of $90^{\circ}$ or below) diverged from simulations.

The total length of the QSP is

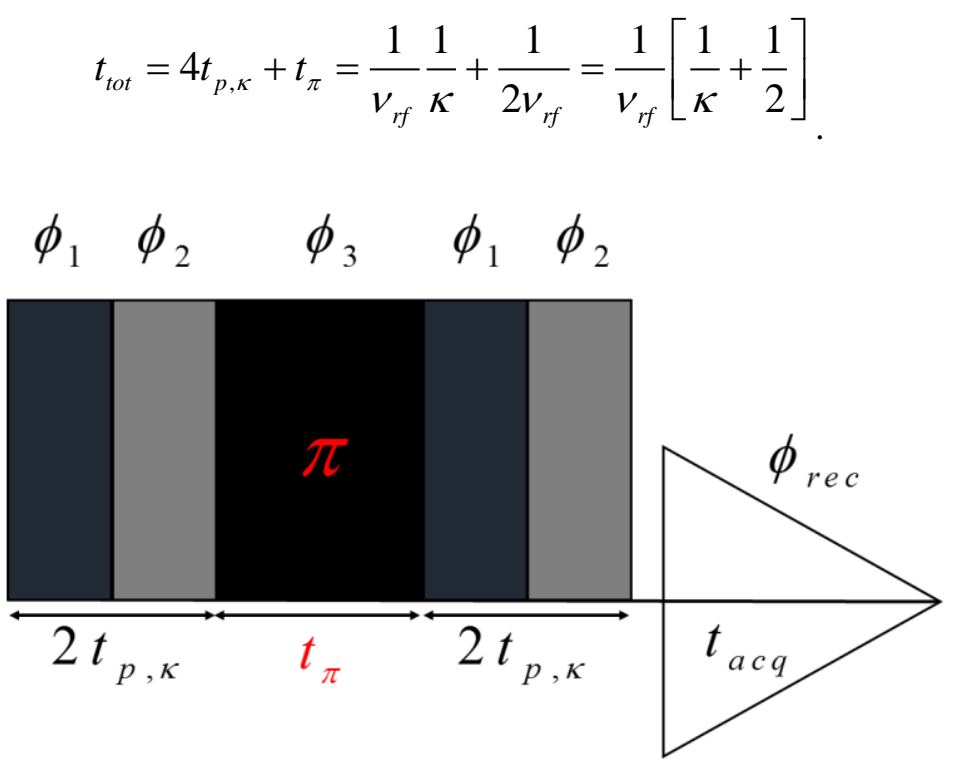

Fig. 2 The QSP sequence consisting of 5 pulses: a $\pi$-pulse in the middle and four pulses with the same lengths $t_{p, \kappa}$ on both sides of the $\pi$-pulse. The phases of the pulses are: $\phi_{1}=0^{0} ; \phi_{2}=180^{0} ; \phi_{3}=0^{0}, 180^{\circ} . \phi_{\text {rec }}=270^{0}$. 
As will be shown further, the value $\kappa$ can be used for scaling the duration of the sequence. Acquiring the signal as a function of this scaling parameter, one can obtain the value of the quadrupolar frequency and an estimate of the quadrupolar coupling distribution.

\section{Experimental methods}

\section{Calculations and Simulations:}

All simulations were performed using MATLAB. Powder integration was performed using 50 angles according to the $\mathrm{ZCW}$ algorithm ${ }^{27}$. Since we considered the behavior of the $C T$ only, $50 \beta$ angles were sufficient.

\section{Materials:}

Pf1 bacteriophage was purchased from ASLA Biotech and was divided among three samples: sample I was pure; samples II and III were diluted with $\mathrm{NaCl}$ solution $(0.4 \mathrm{M})$.

Bovine patellar cartilage was received from Dickson's Farmstand Meats (NYC). The cartilage were cut with a scalpel into the strips. The strips were incubated in phosphate buffered saline (PBS) for 3 hours and were afterwards arbitrarily placed into a quartz tube (sample IV) or a glass tube (sample V). The tubes were filled with fluorinated oil solution $(\mathrm{FC}-84)$ to minimize susceptibility artifacts. After acquiring spectra from sample $\mathrm{IV}, \mathrm{NaCl}$ solution was added above the fluorinert solution (fluorinert is heavier than water and hydrophobic). The aim of the adding of $\mathrm{NaCl}$ solution was to prove experimentally the insensitivity of the QSP to the atoms without quadrupolar coupling.

NMR experiments:

$90^{\circ}$ and QSP experiments were performed using a broad-brand direct observe probe tuned to sodium on a Bruker Avance, $500 \mathrm{MHz}$ spectrometer. Nutation frequencies for ${ }^{23} \mathrm{Na}$ NMR were calibrated using a 0.4 $\mathrm{M}$ solution of $\mathrm{NaCl}$. Table 1 lists the experimental parameters used.

Table 1. Experimental parameters used in the $90^{\circ}$ and QSP experiments.

\begin{tabular}{|c|c|c|c|c|}
\hline Materials & $\mathrm{NS}$ & $\mathrm{SW}^{1}(\mathrm{kHz})$ & $\mathrm{AQ}^{2}(\mathrm{~s})$ & $\mathrm{D}^{3}(\mathrm{~s})$ \\
\hline $\mathrm{NaCl}$ & 64 & 25 & 0.15 & 1.2 \\
\hline $\mathrm{Pf} 1$ & 512 & 25 & 0.15 & 1.2 \\
\hline Cartilage & 512 & 25 & 0.05 & 1.0 \\
\hline
\end{tabular}

${ }^{1}$ Spectral Width; ${ }^{2}$ Acquisition time; ${ }^{3}$ Recycle delay.

\section{Simulations}

In this section we show the excitation of the ordered spins with a single quadrupolar coupling, or a distribution of quadrupolar couplings under with different settings of $v_{r f}, v_{Q}, \kappa$. We also show here the influence of the inhomogeneities of the rf-field and the external field (the model of inhomogeneity is uniform distribution in both cases) on the excitation of these atoms, and how the nature of the quadrupolar coupling distribution can be investigated.

\section{Single quadrupolar coupling frequency}

In Fig. 3 we show the dependence of the $C T$ on $\kappa$ (Eq. (8)) for different values of the quadruple frequency $[0: 1500](\mathrm{Hz})$ for parallel orientation (Eq. (2)); $v_{r f}(\mathrm{~Hz})=1200$ (a), 500 (b) 100 (c). At $v_{Q}=0$ the $C T$ 
does not depend on the values of $v_{r f}, \kappa$ and equals zero. From all three Figures we can see that with increasing quadrupolar frequency the intensity of the $C T$ grows also. From Figs. $3 \mathrm{~b}$ and Fig. $3 \mathrm{c}$ we can see that the behavior of the $C T$ depends on $\kappa$ and the ratio $v_{Q} / v_{r f}$. For example, the absolute value of the $C T$ (the absolute value of the complex signal) for some $\kappa$ when $v_{Q}=1500, v_{r f}=500$ (Fig. 3b, the last red thick peaks) and $v_{Q}=300, v_{r f}=100$ (Fig. 3c, second green thin peak) are the same. The difference between them is the influence of $T_{2}$ relaxation during the pulse. In the insert of that figure we show two spectra of a 3/2 spin with $1500 \mathrm{~Hz}$ quadrupolar frequency which were obtained with an ideal $90^{\circ}$ pulse (black line) and QSP (red line) under the conditions $v_{r f}=500, \kappa=2,\left(v_{Q} / v_{r f}=3\right)$. The intensities of the $C T$ in both cases are the same. When $v_{Q}>6 v_{r f}$ the influence of the quadrupole interaction during the pulse decreases and the intensities of the $C T$ are small (Fig. 5, thick peaks). From this Figure we can conclude that during the QSP there appear terms of first rank in-phase single quantum $\left(T_{1, \pm 1}\right)$ and third rank anti-phase single quantum $\left(T_{3, \pm 1}\right)$ coherences $^{28,29}$. If $v_{Q}<v_{r f}$ the first rank coherences contribute the most to the signal, regardless of $\kappa$. For example, the first four columns of peaks in Fig. $3 \mathrm{~b}$ have positive signs. In the regime where $v_{Q} \geq v_{r f}$ the contribution of the third rank coherences grows and can be larger than the in-phase terms. For example, the last two columns of peaks have negative signs. Consequently, the satellite transitions are anti-phase with respect to $C T$ (insert of Fig. 3b).

As described above, QSP can be used for excitation of ordered spins and can be used for obtaining the values of the quadrupolar frequencies also. Under the condition $v_{Q}<v_{r f}$ the behavior of the $C T$ for different $\kappa$ is similar. It can clearly be seen in Fig. 3a for three couplings of $v_{Q}=150 ; 300 ; 450 \mathrm{~Hz}$ and in Fig. $3 \mathrm{~b}$ for two couplings with $v_{Q}=150 ; 300 \mathrm{~Hz}$. In the insert of Fig. 3a we show the behaviors of the $C T$ as a function of $\kappa$ for $v_{Q}=150 \mathrm{~Hz}$ (black curve), $v_{Q}=450 \mathrm{~Hz}$ (red curve), and $v_{Q}=600 \mathrm{~Hz}$ (black curve with circles). The points are normalized by the intensity of the first point, which has the largest intensity, as represented by

$$
\overline{C T}\left(v_{Q} ; \kappa\right)=C T\left(v_{Q} ; \kappa\right) / C T_{\max }\left(v_{Q} ; \kappa=1.25\right) .
$$

If the curve with $v_{Q}=150 \mathrm{~Hz}$ and the curve with $v_{Q}=450 \mathrm{~Hz}$ are hardly distinguished, the curve with $600 \mathrm{~Hz}$ quadrupolar frequency can be distinguished from the curve with $450 \mathrm{~Hz}$ of quadrupolar frequency (Fig. 3a, insert), but the quadrupolar frequency resolution is low. When increasing the ratio of $v_{Q} / v_{r f}$, the quadrupolar frequency resolution decreases. The quadrupolar frequency resolution means the minimal value $\delta v_{Q}$, when two curves $C T\left(v_{Q} ; \kappa\right)$ and $C T\left(v_{Q}+\delta v_{Q} ; \kappa\right)$ can be distinguished. Under some experimental conditions this value can be about $\delta v_{Q} \approx 2-5 \mathrm{~Hz}$. 


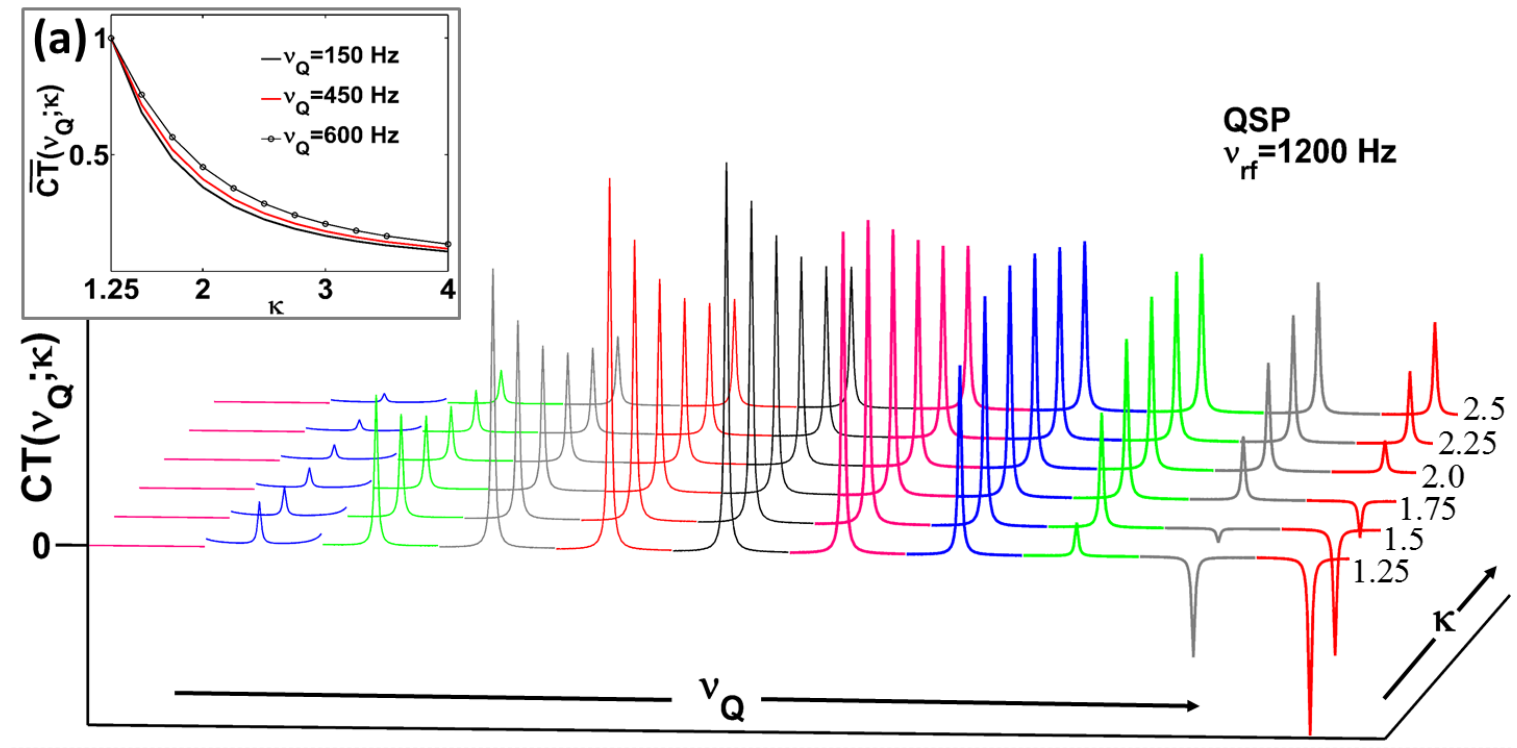

(b)

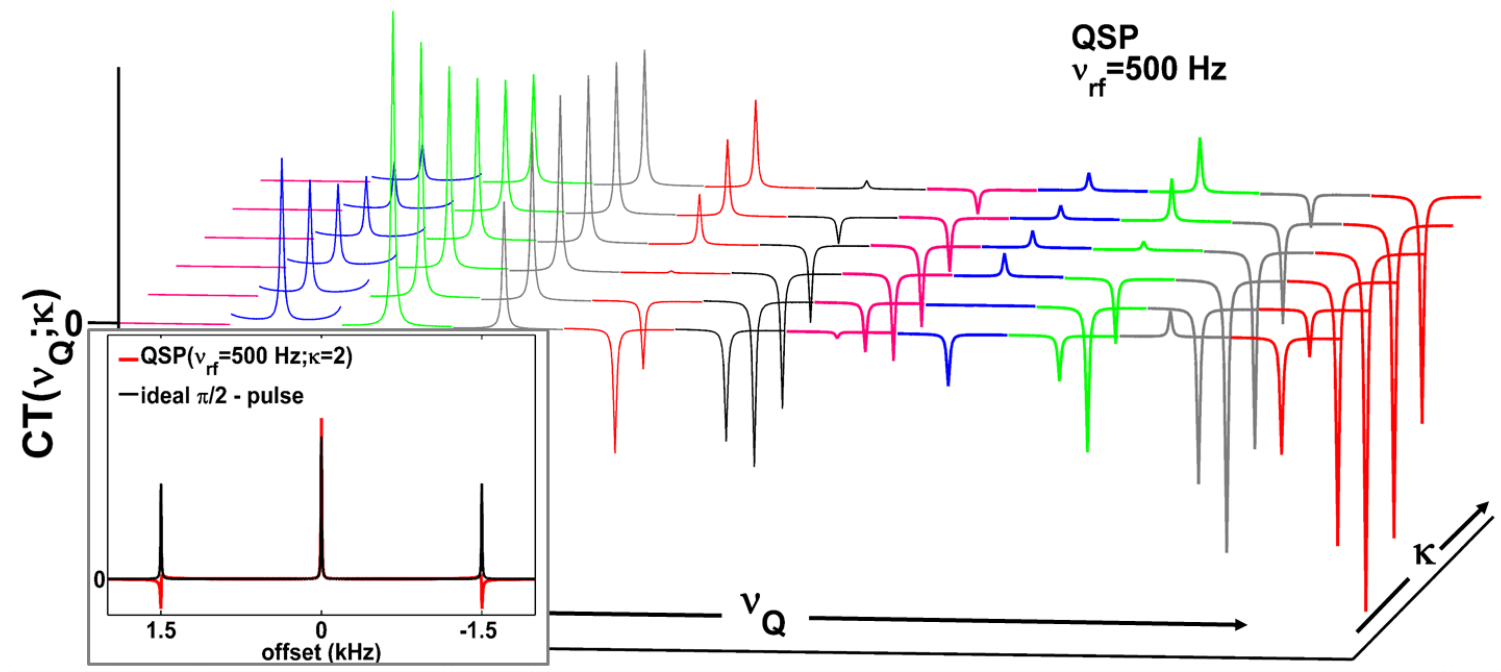

(c)

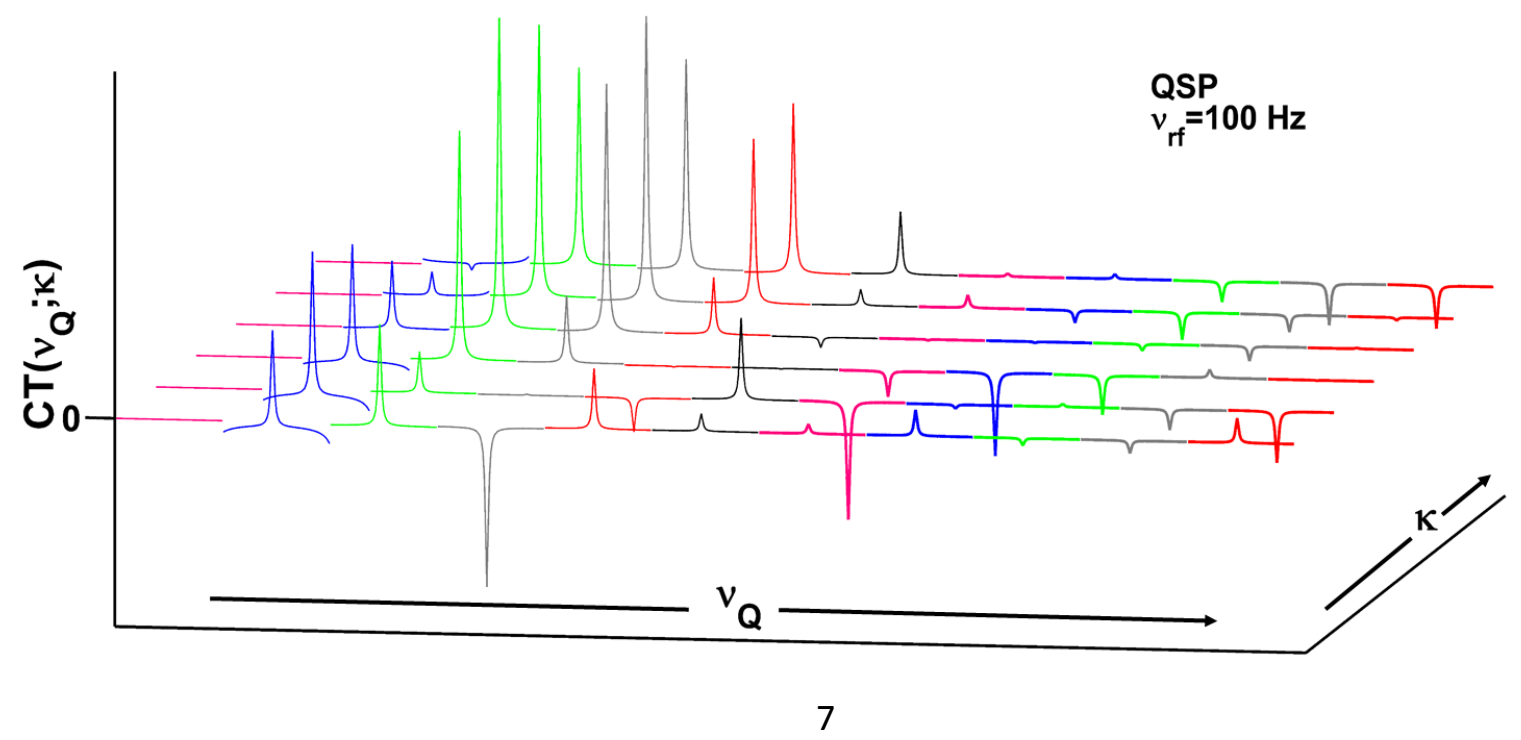


Fig. 3 The dependence of the $C T$ on $\kappa[1.25 ; 1.5 ; 1.75 ; 2 ; 2.25 ; 2.5]$ (y-axis) and $v_{Q}(\mathrm{~Hz})[0 ; 150 ; 300 ; 450 ; 600 ; 750 ; 900$; 1050; 1200; 1350; 1500] (x-axis) at different values of $v_{r f}$. (a): $v_{r f}=1200 \mathrm{~Hz}$. Insert: QSP curves: $v_{Q}=150 \mathrm{~Hz}$ (black line); $v_{Q}=450 \mathrm{~Hz}$ (red line); $v_{Q}=600 \mathrm{~Hz}$ (black line with circles); (b): $v_{r f}=500 \mathrm{~Hz}$. Insert: red - QSP spectrum under conditions $v_{r f}=500 \mathrm{~Hz}, v_{Q}=1500 \mathrm{~Hz}, \kappa=2$; black - spectrum of $1500 \mathrm{~Hz}$ quadrupolar frequency after ideal $90^{0}-$ pulse; (c): $v_{r f}=$ $100 \mathrm{~Hz}$.

Inhomogeneity of $\mathrm{B}_{0}$ could produce some residual signal of atoms without quadrupolar couplings from the QSP sequence. In Fig. $4 \mathrm{a}$ we show the influence of $\mp 20 \mathrm{~Hz}$ of $\Delta B_{0}$ on a $S=3 / 2$ spectrum with $v_{Q}=0$ (black dotted line) and $S=3 / 2$ spectrum with $v_{Q}=400 \mathrm{~Hz}$ (red thick line). Also we show the spectrum without inhomogeneity (blue thin line) for comparison.
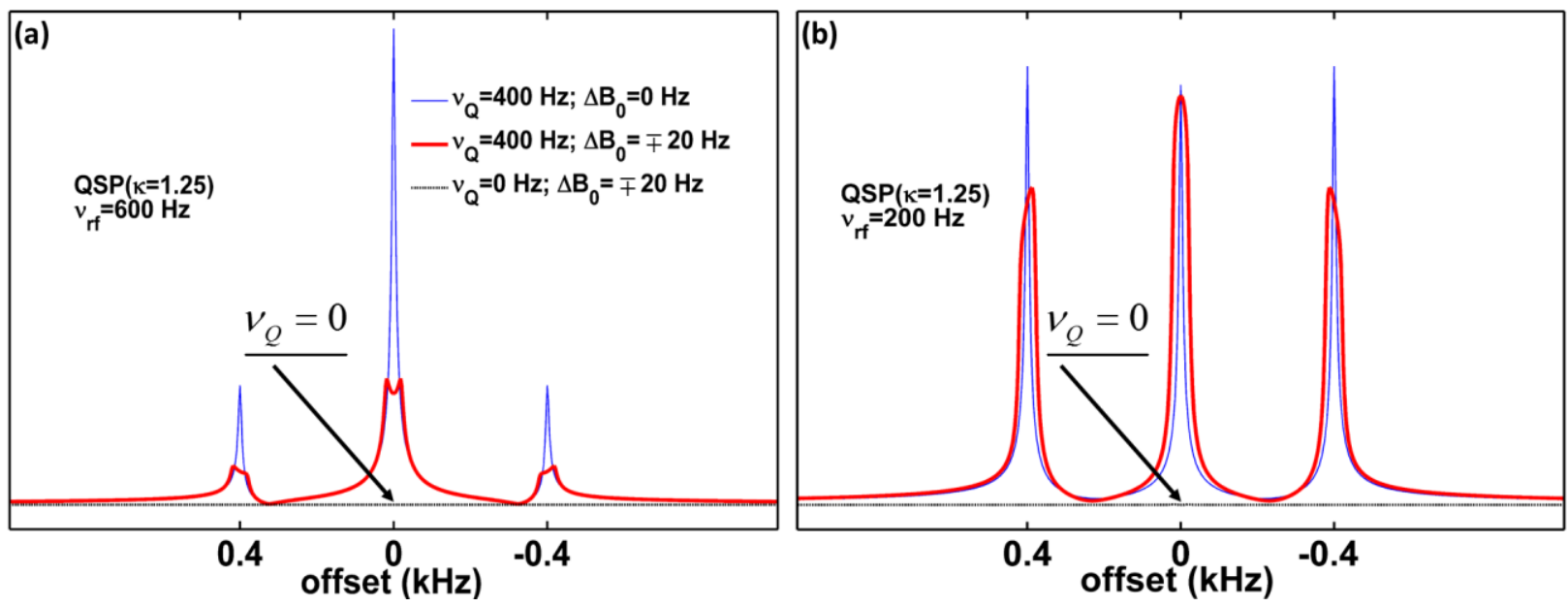

Fig. 4 The dependence of the $C T(\kappa=1.25)$ on the inhomogeneity of the external field $\left(\Delta B_{0}\right)$ for different rf - field strengths: (a) $v_{r f}=600 \mathrm{~Hz}$; (b) $v_{r f}=200 \mathrm{~Hz}\left(v_{Q} / v_{r f}=3\right)$. Blue thin spectrum $-v_{Q}=400 \mathrm{~Hz}, \Delta B_{0}=0$; red thick spectrum $-v_{Q}=400 \mathrm{~Hz}$, $\Delta B_{0}(H z) \in[-20: 20]$; black dotted spectrum $v_{Q}=0, \Delta B_{0}(H z) \in[-20: 20]$. The intensity of the blue peaks in (a) is about 10 times larger than blue peaks in (b).

It is seen that under the conditions investigated, the inhomogeneity has a negligible influence on isotropic spins (black dotted spectrum). On the other hand, it has a significant influence on the signals from ordered spins: the value of $C T$ under inhomogeneity is smaller by about a factor of 4 compared to $C T$ without inhomogeneity. Interestingly, using the very weak rf-fields such as $200 \mathrm{~Hz}$ one can significantly decrease the influence of $\Delta B_{0}$ (Fig. 4b). This effect occurs because the intensity of the blue peak in Fig. $4 \mathrm{~b}$ has no maximum value (the intensity of the blue peaks in (a) is about 10 times larger than the blue peaks in (b)). In the presence of inhomogeneity, the intensity of $C T$ is a little increased for certain offsets, and at other offsets it is decreased, but the intensity of the total signal is not changed.

The inhomogeneity of the rf-field $\Delta v_{r f}$ does not excite isotropic spins because of the structure of QSP, but it influences the signal of the spins when quadrupolar coupling is present and the ability of obtaining accurate measures of the quadrupolar frequency. In Fig. 5 we show the influence of $10 \%$ of $\Delta v_{r f}$ (red thick lines) on $C T\left(v_{Q} ; \kappa=1.25\right)$ for $v_{r f}=200 \mathrm{~Hz}(\mathrm{a}, \mathrm{b})$ and $v_{r f}=500 \mathrm{~Hz}(\mathrm{c}, \mathrm{d})$ and compare it with $C T\left(v_{Q} ; \kappa=1.25\right)$ without inhomogeneity (blue thin lines). In the inserts of these Figures we show the behavior of the $C T$ as a function of $\kappa$ with $\Delta v_{r f}$ and without, respectively. When $v_{r f}<v_{Q}$ like in Fig. 5a and in Fig.5c, the $\Delta v_{r f}$ has a significant influence on $C T\left(v_{Q} ; \kappa\right)$. In the first case, inhomogeneity leads to $45 \%$ of reduction of the $C T$ and in the second it leads to an increase by $130 \%$. 
On other hand, it is important to point out that $\Delta v_{r f}$ does not have a significant influence on $\overline{C T}\left(v_{Q} ; \kappa\right)$ curves, except on the first points $\overline{C T}\left(v_{Q}=600 \mathrm{~Hz} ; \kappa=1.25\right)$ (insert, Fig.5a) and $\overline{C T}\left(v_{Q}=1200 \mathrm{~Hz}\right.$; $\kappa=1.25$ ) (insert, Fig.5c). When $v_{r f}>v_{Q}$ like in Fig. 5b and $v_{r f} \approx v_{Q}$ in Fig.5d, $\Delta v_{r f}$ has a very small influence on $C T$ and on the $\overline{C T}\left(v_{Q} ; \kappa\right)$ curves, accordingly. Therefore $\mathrm{B}_{1}$ inhomogeneity will not have a significant influence on the ability of obtaining the quadrupolar frequencies.
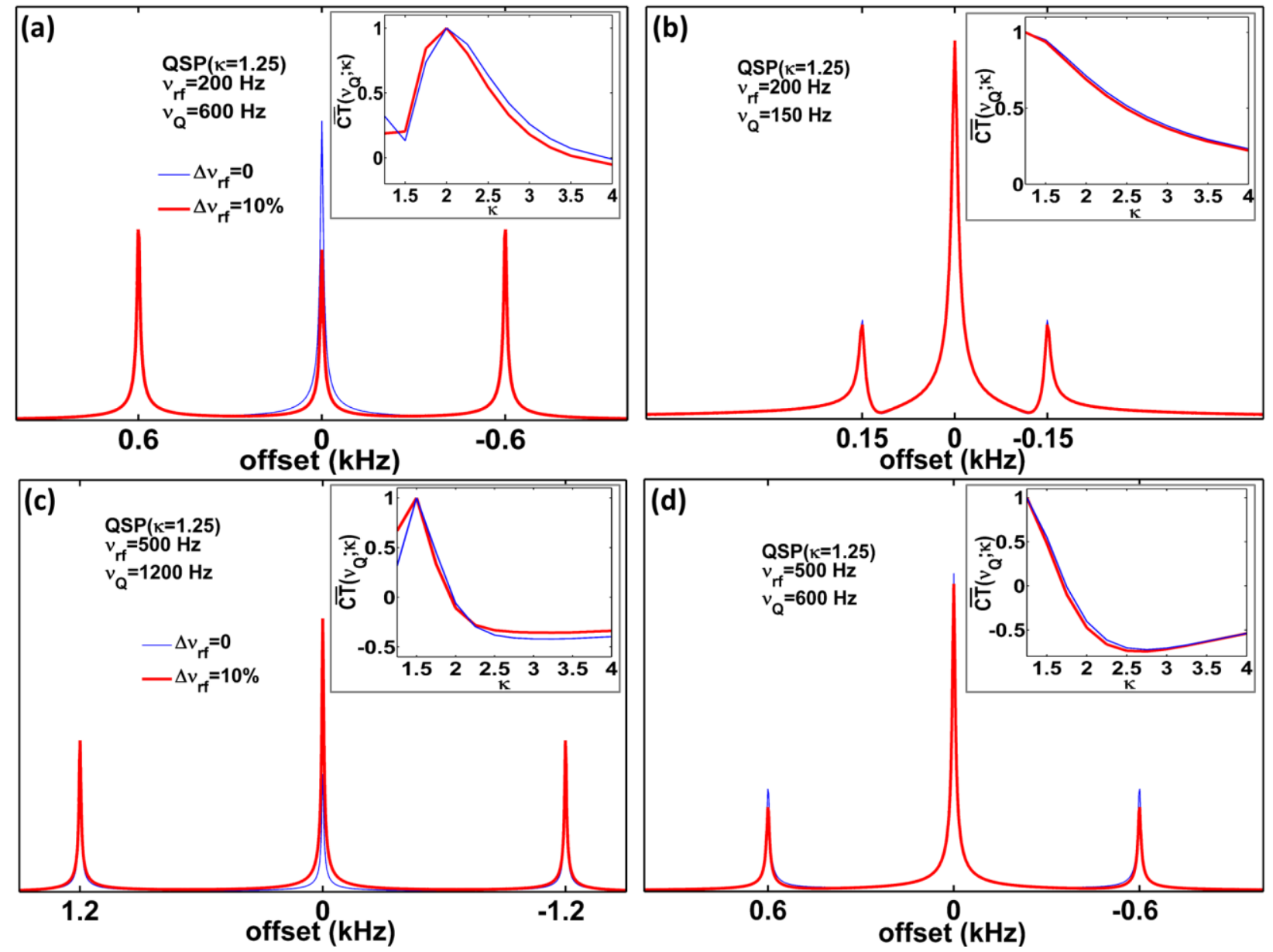

Fig. 5 The behaviors of the $C T(\kappa=1.25)$ and $\overline{C T}\left(v_{Q} ; \kappa\right)$ (inserts) without (blue line) and with (red line) inhomogeneity of the rf - field $\left(\Delta v_{r f}\right):$ (a) $v_{r f}=200 \mathrm{~Hz}, v_{Q}=600 \mathrm{~Hz}$; (b) $v_{r f}=200 \mathrm{~Hz}, v_{Q}=150 \mathrm{~Hz}$; (c) $v_{r f}=500 \mathrm{~Hz}, v_{Q}=1200 \mathrm{~Hz}$; (d) $v_{r f}=500 \mathrm{~Hz}$, $v_{Q}=600 \mathrm{~Hz}$.

\section{Distribution of quadrupolar couplings}

The behavior of the $C T$ of a powder follows the broad trends of the $C T$ with a single coupling. In Figs. 6 and 7 we show the dependence of the absolute value of $C T$ on $\kappa$ for different values of quadrupolar frequency [25:1000] (Hz), $v_{r f}=100 \mathrm{~Hz}$ (Fig. 6) and [200:4600] (Hz), $v_{r f}=600 \mathrm{~Hz}$ (Fig. 7). Like in the case of a single quadrupolar coupling frequency, during the QSP there appear terms of first rank in-phase single quantum $\left(T_{1, \pm 1}\right)$ and third rank anti-phase single quantum $\left(T_{3, \pm 1}\right)$ coherences. Under $v_{Q} \lesssim 2 v_{r f}$ the first rank coherences contribute the most to the signal (in Figs. 6 and 7, the first four couplings $v_{Q}=25,50,100,200 \mathrm{~Hz}$ and $v_{Q}=200,600,1000,1400 \mathrm{~Hz}$, respectively). Under $v_{Q} \gtrsim 5.5 v_{r f}$ the 
third rank coherences contribute the most to the signal (in Figs. 6 and 7, the last five couplings $v_{Q}=$ $600,700,800,900,1000 \mathrm{~Hz}$ and $v_{Q}=3000,3400,3800,4200,4600 \mathrm{~Hz}$, respectively). Between these two conditions, the contributions of the first and third rank coherences are approximately equal and therefore the intensities are small (in Figs. 6 and 7, the middle three couplings $v_{Q}=300,400,500 \mathrm{~Hz}$ and $v_{Q}=1800,2200,2600 \mathrm{~Hz}$, respectively).A dependence of $\overline{C T}$ on $v_{Q}$ is seen under the conditions $v_{Q} \geq v_{r f}$ (insert of Fig. 6) and the maximum of the intensity of $C T$ under the conditions $v_{Q} / v_{r f}=1.25$ and $v_{Q} / v_{r f}=7.6$ can be achieved at an intensity of $58 \%$ and $29 \%$ of the original one only (insert of Fig. 7).

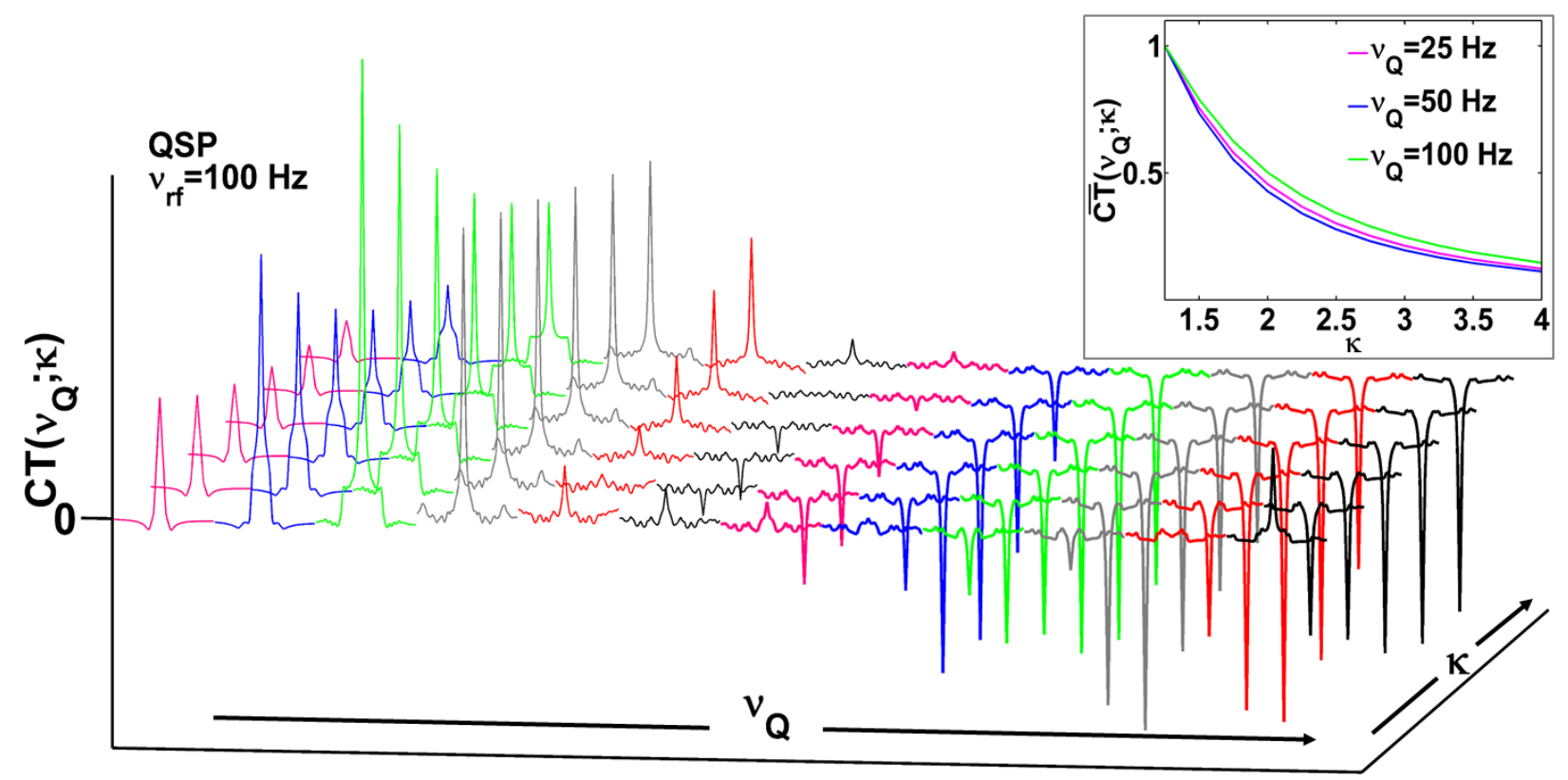

Fig.6 The dependence of the $C T$ at $v_{r f}=100 \mathrm{~Hz}$ as function of $\kappa[1.25 ; 1.5 ; 1.75 ; 2 ; 2.25 ; 2.5]$ (y-axis) and $v_{Q}(\mathrm{~Hz})[25 ; 50$; 100; 200; 300; 400; 500; 600;700;800; 900; 1000] (x-axis). Insert: QSP curves: $v_{Q}=25 \mathrm{~Hz}$ (magenta line); $v_{Q}=50 \mathrm{~Hz}$ (blue line); $v_{Q}=100 \mathrm{~Hz}$ (green line). 


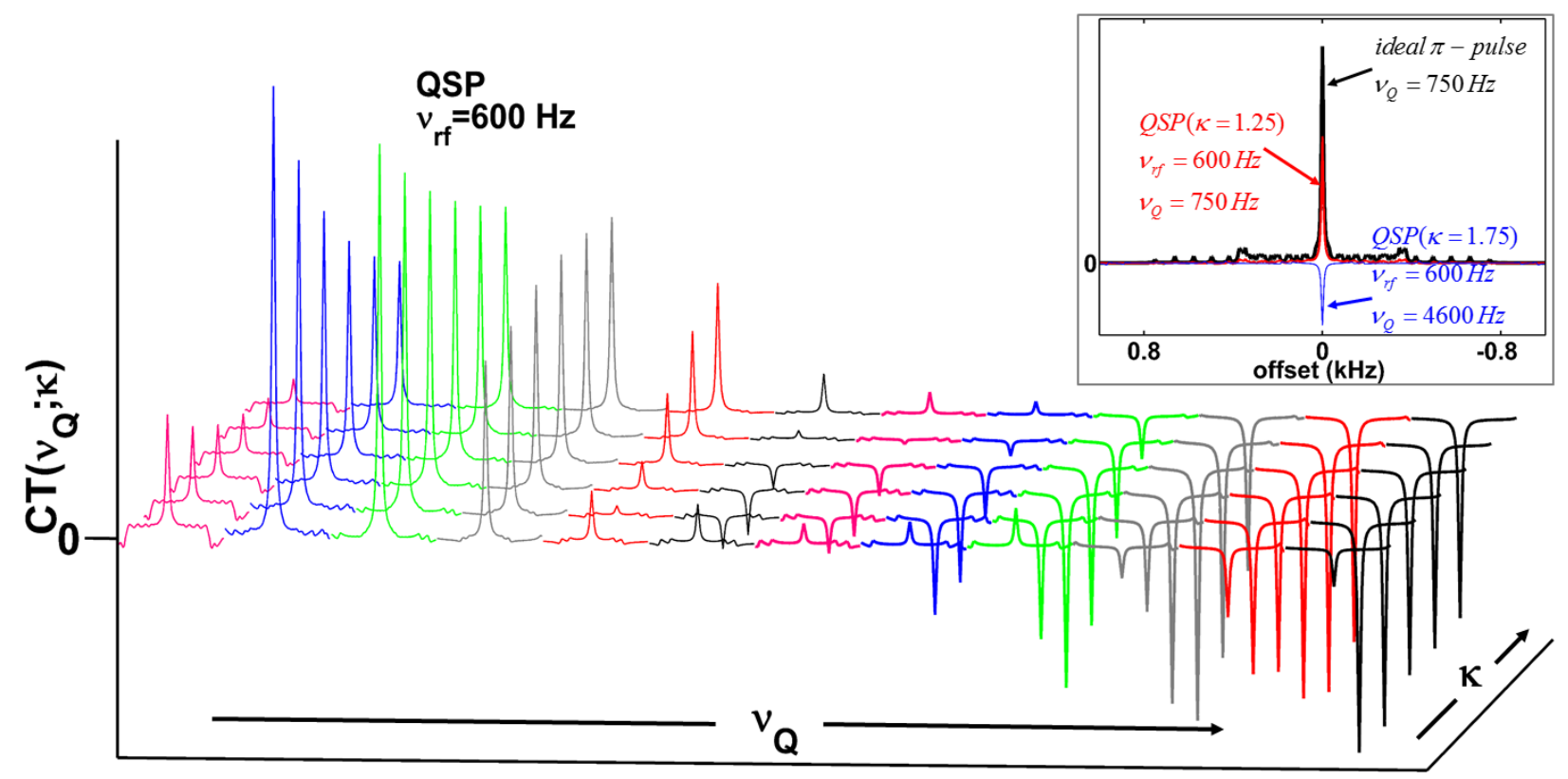

Fig. 7 The dependence of the $C T$ at $\left(v_{r f}=500 \mathrm{~Hz}\right)$ as a function of $\kappa[1.25 ; 1.5 ; 1.75 ; 2 ; 2.25 ; 2.5]$ (y-axis) and $v_{Q}(\mathrm{~Hz})[200$; $600 ; 1000 ; 1400 ; 1800 ; 2200 ; 2600 ; 3000 ; 3400 ; 3800 ; 4200 ; 4600]$ (x-axis). Insert: black - spectrum of 750 Hz quadrupolar frequency after ideal $90^{\circ}-$ pulse; red - QSP spectrum under conditions $v_{r f}=600 \mathrm{~Hz}, v_{Q}=750 \mathrm{~Hz}, \kappa=1.25$; blue- QSP spectrum under conditions $v_{r f}=600 \mathrm{~Hz}, v_{Q}=4600 \mathrm{~Hz}, \kappa=1.75$. The ratio of intensities is 1:0.58:0.29.

In Fig. 8 we show the influence of $10 \%$ of inhomogeneity of rf-field $\left(\Delta v_{r f}\right.$ in the case when $v_{Q}<v_{r f}$ (Fig. 8a) and in the case when $v_{Q}>v_{r f}$ (Fig. 8b and c). In the first case, the influence of the inhomogeneity is negligible. In the case where $v_{r f}=600 \mathrm{~Hz}, v_{Q}=1200 \mathrm{~Hz}$ (Fig. 10b), the inhomogeneity has only a very small influence on the curve $\overline{C T}\left(v_{Q} ; \kappa\right)$ (insert of Fig. 10b) and can lead to a small error in the extracted quadrupolar frequency (about 20-30 Hz). In the third case (Fig. 10c), the inhomogeneity has significant influence on the $C T\left(v_{Q} ; \kappa\right)$. As in the case of the single quadrupolar coupling, $\Delta v_{r f}$ has a moderate influence on $\overline{C T}\left(v_{Q} ; \kappa\right)$ curves on the first points only (insert of Fig. 10c).
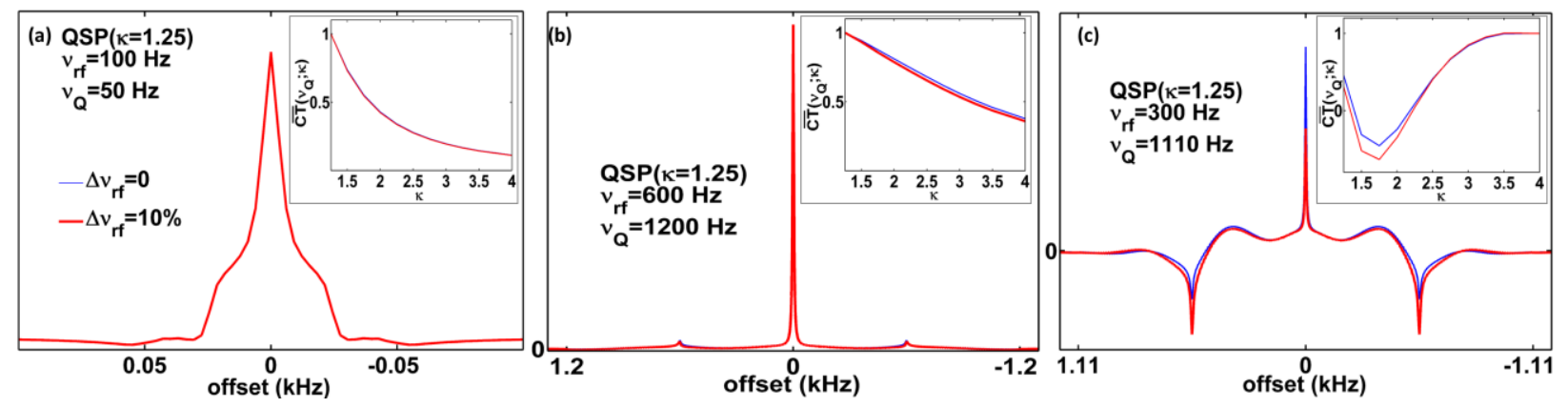

Fig. 8 The behaviors of the $C T(\kappa=1.25)$ and $\overline{C T}\left(v_{Q} ; \kappa\right)$ (inserts) without (blue line) and with (red line) inhomogeneity of the rffield $\left(\Delta v_{r f}\right)$ : (a) $v_{r f}=100 \mathrm{~Hz}, v_{Q}=50 \mathrm{~Hz}$; (b) $v_{r f}=600 \mathrm{~Hz}, v_{Q}=1200 \mathrm{~Hz}$; (c) $v_{r f}=300 \mathrm{~Hz}, v_{Q}=1110 \mathrm{~Hz}$. The number of orientations which was used in (a) is 50 and 538 in the case of (b) and (c). For obtaining $\overline{C T}\left(v_{Q} ; \kappa\right)$ curves the number of orientations was 50 .

As mentioned above, using this method we can investigate the nature of the quadrupolar coupling distribution. We focus here on two models: uniform orientational distribution (Eq. (4)) or Gaussian quadrupolar coupling distribution (Eq. (5)). The last figure of this section show the dependence of 
$\overline{C T}\left(v_{Q}^{\text {loc }}=1000 \mathrm{~Hz} ; \kappa\right)$ on the width of the quadrupolar distribution $\Delta v_{Q}^{\text {loc }}[0 ; 50 ; 100 ; 150 ; 200 ; 250 ; 300]$ under different values of $v_{r f}(\mathrm{~Hz}): 600$ (a); 500 (b); 400 (c). Under $600 \mathrm{~Hz}$ of rf-field strength (Fig. 9a) $\overline{C T}\left(v_{Q}^{l o c}, \Delta v_{Q}^{l o c} ; \kappa\right)$ has a very small dependence on $\Delta v_{Q}^{\text {loc }}$. Under $500 \mathrm{~Hz}$ of rf-field strength (Fig. 9b) this dependence grows and under $400 \mathrm{~Hz}$ of rf-field (Fig. 9c) there is a visual difference of $\overline{C T}\left(v_{Q}^{l o c}=1000 \mathrm{~Hz} ; \kappa \leq 2.5\right)$ for different $\Delta v_{Q}^{l o c}$. Therefore, the experiments, in which the first point $C T\left(v_{Q} ; \kappa=1.25\right)$ has the maximum value (Fig. 9a and b) and the curves drop continuously, can be investigated with a model of a random distribution of principal axis frames (Eq. (4)) for obtaining the value of $v_{Q}$. Other experiments, where the behaviors of the QSP curves are more complicate (for example, like in Fig. 9c) can be used for investigation of the nature of the quadrupolar coupling distribution.
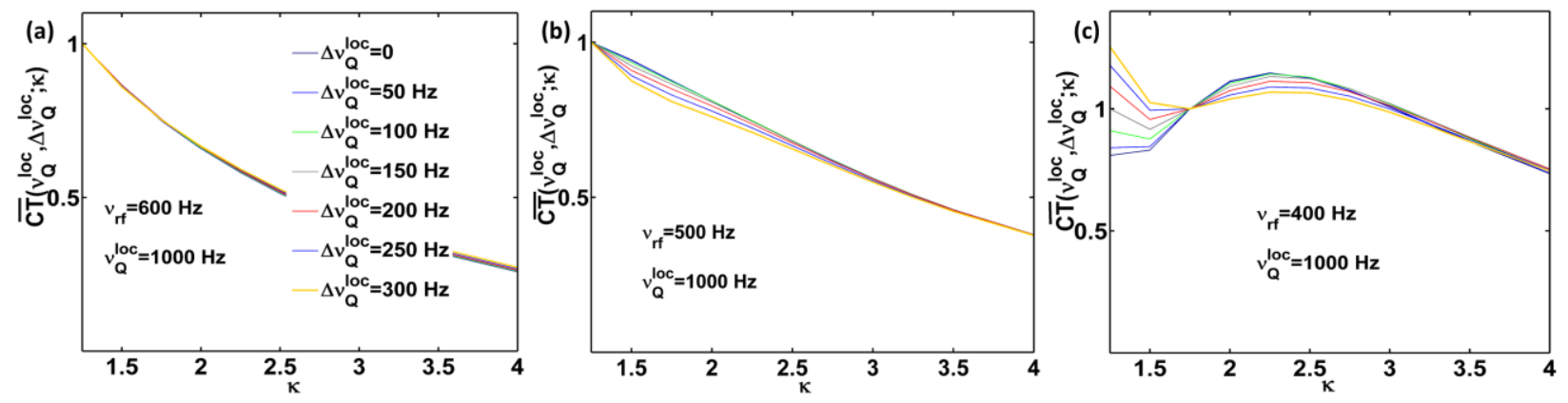

Fig. 9 The dependence of $\overline{C T}\left(v_{Q} ; \kappa\right)$ on $\Delta v_{Q}^{\text {loc }}=0,50,100,150,200,250,300 \mathrm{~Hz}:$ (a) $v_{r f}=600 \mathrm{~Hz}$; (b) $v_{r f}=500 \mathrm{~Hz}$; (c) $v_{r f}=400 \mathrm{~Hz} . v_{Q}^{\text {loc }}=1000 \mathrm{~Hz}$.

In this section we showed that QSP excites the ordered spins with non-vanishing quadrupolar coupling only. When $v_{r f} \leq v_{Q}$ the $C T$ has a different behavior as function of $\kappa$ when the quadrupolar frequencies differ, and therefore, the value of the quadrupolar frequency can be extracted from these curves. When $v_{r f} \lesssim 2.5 v_{Q}$ the $C T$ curves begin to depend on the nature of the quadrupolar coupling distribution also and therefore, these can also be characterized by QSP experiments. The moderate inhomogeneity of the external field does not influence isotropic spins, but it has a strong influence on the excitation of the ordered spins. The moderate inhomogeneity of the rf-field does not influence the isotropic spins significantly and does not influence the ability to determine the values of quadrupolar frequencies of the ordered spins. As shown here, in experiments where the curves drop continuously, one can use a uniform orientational model, When the behavior of the QSP curves are more complicated (for example, Fig. 9c) one can use a Gaussian quadrupolar coupling distribution.

\section{Experimental results}

We now turn to experiments where we explore the properties of the QSP experiments for their ability to selectively observe odered sodium and characterize the quadrupolar coupling distributions. Here, we use samples ${ }^{23} \mathrm{Na}$ in $\mathrm{Pf} 1$, and in cartilage and contrast that to the behavior of isotropic sodium in $\mathrm{NaCl}$ solution. Further experiments are shown, which demonstrate that the quadrupolar couplings can be characterized even in the presence of strong background signals from glass tubes. 
In Fig. 10, we show ${ }^{23} \mathrm{Na}$ spectra of a $\mathrm{NaCl}$ solution $(0.4 \mathrm{M})$, which demonstrate that the QSP does not excite isotropic spins under different experimental conditions. The black line is the ${ }^{23} \mathrm{Na}$ spectrum after a regular $90^{\circ}$ hard-pulse excitation. All four QSP spectra (color lines) are very small compared to the $90^{\circ}$ spectrum. The ratio of intensities of the $90^{\circ}$ spectrum and QSP spectra are 100:1:1.2:1.5:2.

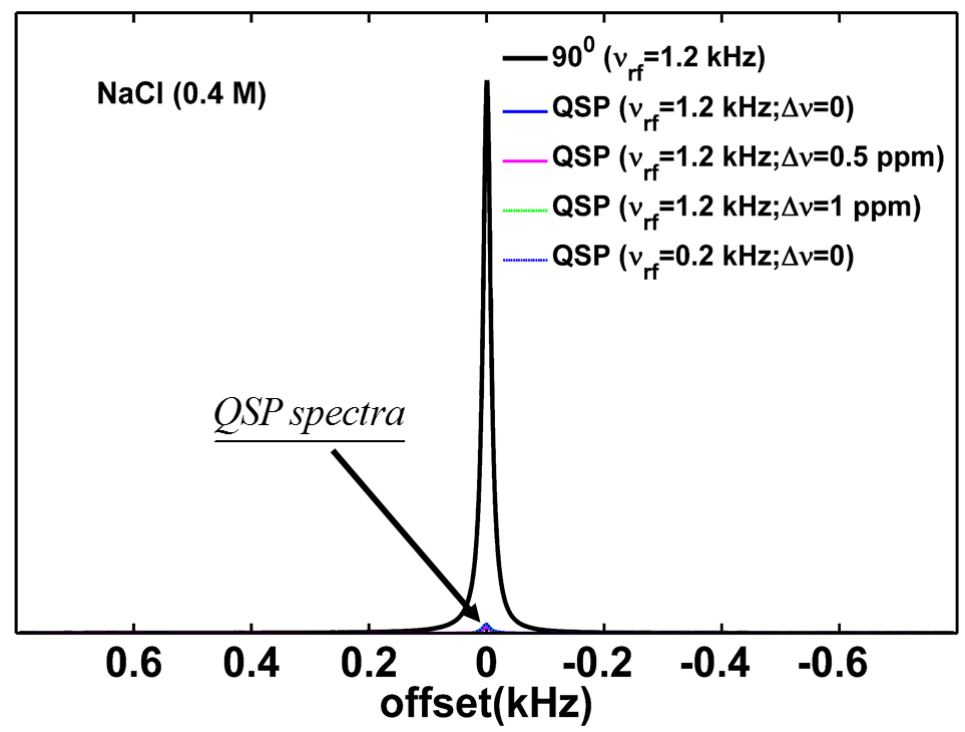

Fig. $10^{23} \mathrm{Na}$ spectrum in $\mathrm{NaCl}(0.4 \mathrm{M})$ solution. Black line $90^{\circ}-$ pulse $\left(v_{r f}=1200 \mathrm{~Hz}\right)$. Other QSP $(\kappa=1.25)$ : blue line $\left(v_{r f}=1200 \mathrm{~Hz}, \Delta v=0\right)$; magenta line $\left(v_{r f}=1200 \mathrm{~Hz}, \Delta v=0.5 \mathrm{ppm}\right)$; green line $\left(v_{r f}=1200 \mathrm{~Hz}, \Delta v=1 \mathrm{ppm}\right)$; blue dotted line $\left(v_{r f}=200 \mathrm{~Hz}, \Delta v=0 \mathrm{ppm}\right)$. The ratio of intensities of these spectra are 100:1:1.2:1.5:2.

In Fig. 11 we show three different $90^{\circ}{ }^{23} \mathrm{Na}$ spectra of Pf1 bacteriophage (a) pure sample (I) and two samples diluted with $\mathrm{NaCl}(0.4 \mathrm{M})$ solution (b) - II, (c) - III. The measured quadrupolar frequency of the first sample (a) is approximately $806 \mathrm{~Hz}$, of the second sample (d) it is approximately $498 \mathrm{~Hz}$ and of the third (c) it is approximately $152 \mathrm{~Hz}$.

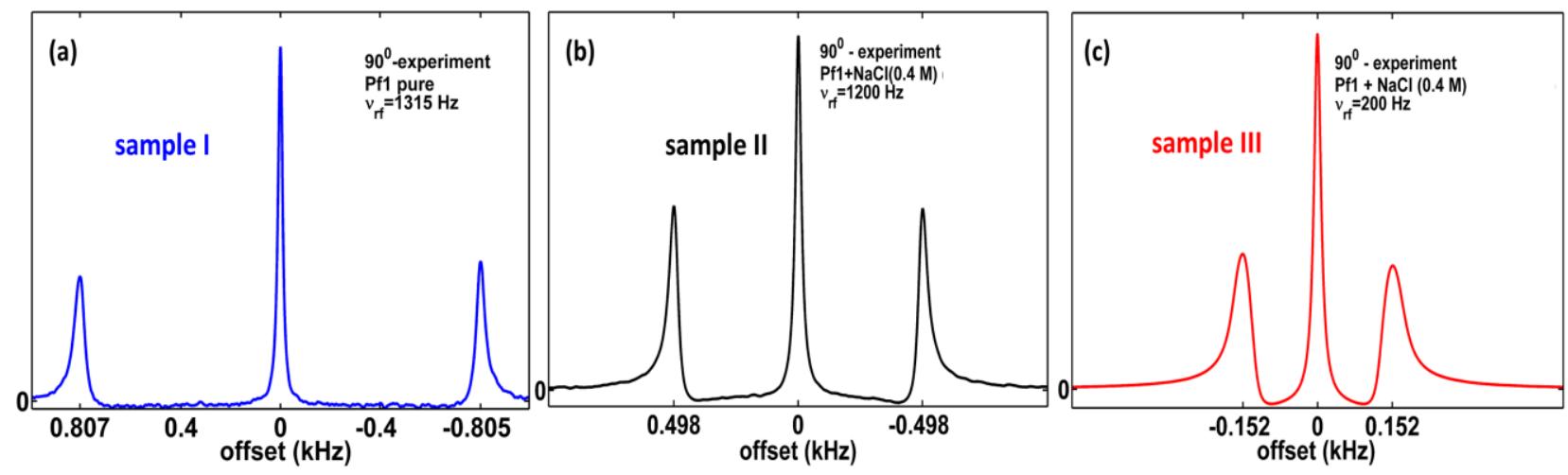

Fig. $11^{23} \mathrm{Na} 90^{0}$ pulse spectra of Pf1 bacteriophage in different concentrations. (a) - pure sample (sample I), $v_{r f}=1315 \mathrm{~Hz}$. Diluted samples with $\mathrm{NaCl}$ : (b) (sample II) $v_{r f}=1200 \mathrm{~Hz}$; (c) (sample III) $v_{r f}=200 \mathrm{~Hz}$.

Fig. 12 shows the results from 6 series of QSP experiments as a function of $\kappa$ : (a-c) sample I; (d-e) sample II; (f) sample III. The obtained quadrupolar frequencies are shown in Table 2. 

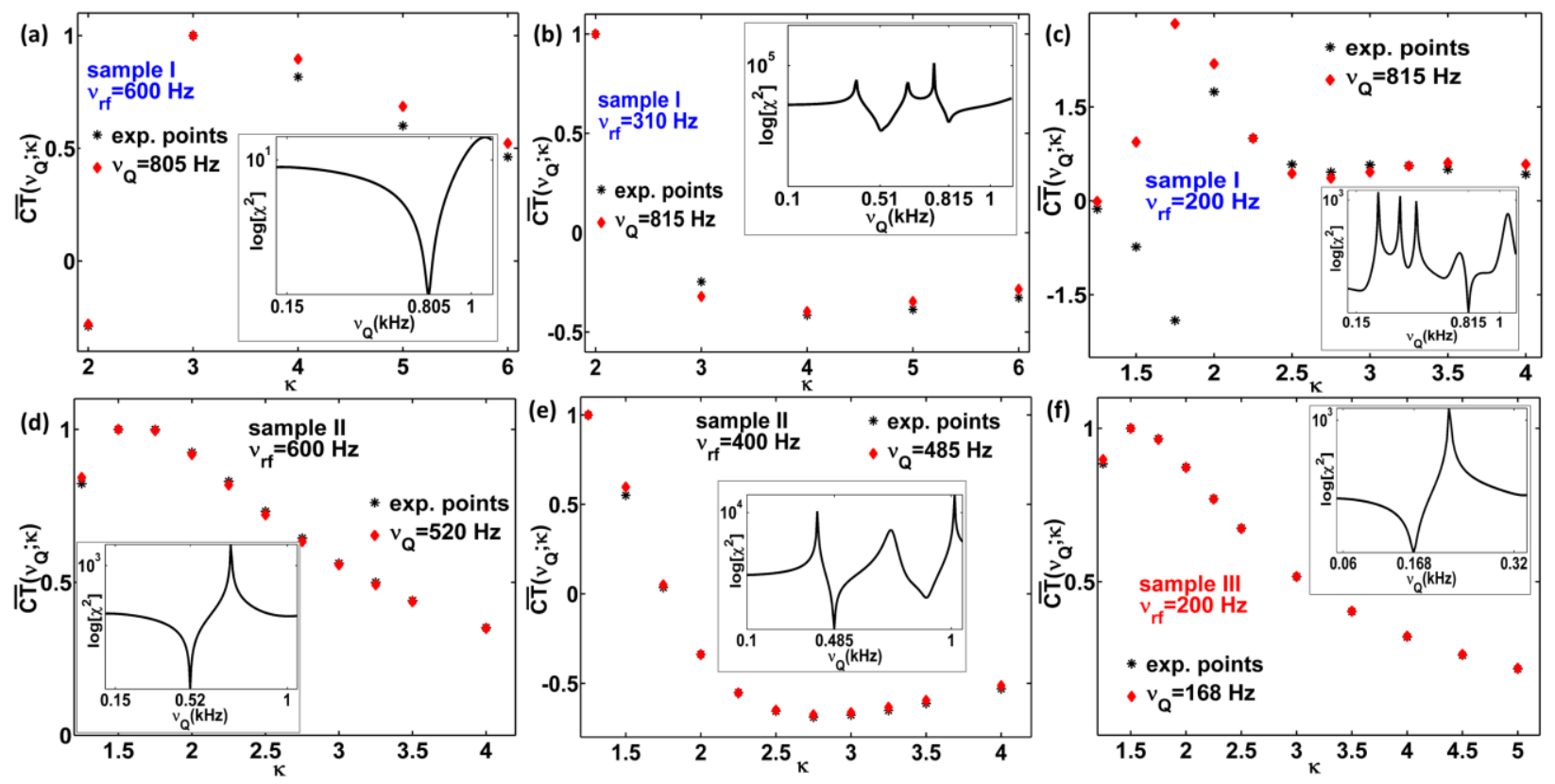

Fig. $12 \overline{C T}\left(v_{Q} ; \kappa\right)$ curves of I sample (a, b, c), sample II (d, e) and sample III (f). (a) $v_{r f}=600 \mathrm{~Hz}$; (b) $v_{r f}=310 \mathrm{~Hz}$; (c) $v_{r f}=200 \mathrm{~Hz}$; (d) $v_{r f}=600 \mathrm{~Hz}$; (e) $v_{r f}=400 \mathrm{~Hz}$; (f) $v_{r f}=200 \mathrm{~Hz}$. The inserts show the logarithm of the residual from least squares fitting (Levenberg-Marquardt method) as function of quadrupolar frequency.

Table 2. The obtained quadrupolar frequencies with direct measurements (spectrum) and QSP experiments with different $v_{r f}$ (Figs.11 and 12).

\begin{tabular}{|c|c|c|c|c|}
\hline sample I & spectrum & $v_{r f}=600 \mathrm{~Hz}$ & $v_{r f}=315 \mathrm{~Hz}$ & $v_{r f}=200 \mathrm{~Hz}$ \\
\hline$v_{Q}(\mathrm{~Hz})$ & 806 & 805 & $510 / 815$ & 815 \\
\hline sample II & spectrum & $v_{r f}=600 \mathrm{~Hz}$ & $v_{r f}=400 \mathrm{~Hz}$ & \\
\hline$v_{Q}(\mathrm{~Hz})$ & 498 & 520 & 485 & \\
\hline sample III & spectrum & $v_{r f}=200 \mathrm{~Hz}$ & & \\
\hline$v_{Q}(\mathrm{~Hz})$ & 152 & 168 & & \\
\hline
\end{tabular}

Five of the six inserts in Fig. 12 (a, c-f) show only one unique minimum of the fitting residual, $\log \left[\chi^{2}\right]$, as function of $v_{Q}$ and they are close to the quadrupolar frequencies which were obtained with the direct measurements. The insert of Fig. 12b shows two minima of $\log \left[\chi^{2}\right]$ at the quadrupolar frequencies of 510 $\mathrm{Hz}$ and $815 \mathrm{~Hz}$. This effect is seen because at $\kappa \geq 4$ the behaviors of $C T\left(v_{Q}=510 \mathrm{~Hz} ; k\right)$ and $C T\left(v_{Q}=815 \mathrm{~Hz} ; k\right)$ are similar. Since the first point serves as a normalized point, only one point $C T\left(v_{Q} ; \kappa=3\right)$ differentiates them. On the one hand, that problem can be solved using smaller values of $\kappa(\kappa=1.25,1.5,1.75)$, because the strongest dependence of $\overline{C T}$ on $\kappa$ is in the range $\kappa \in(1: 2)$. On the other hand, under the condition when the total time of the pulse $\tau_{p} \sim T_{2}$, the relaxation has a strong influence on the magnetization during the pulse and it leads to results which diverge from simulations ${ }^{30}$. The most natural solution of the relaxation problem would be changing the rf-field strength of adjacent pulses and not the total length of QSP by changing $\kappa$. But as Fig. 13 shows, this strategy does not work: the simulation does not fit the experimental points and the obtained quadrupolar frequency, $700 \mathrm{~Hz}$, is far from the directly measured value of $498 \mathrm{~Hz}$. This is an indication that biexponential relaxation, where the 
central and satellite transitions have different relaxation times $T_{2, C T}$ and $T_{2, S T}$ is the reason for the observed effects (Fig. 12c) ${ }^{31}$. . The central transition of the first order Hamiltonian does not depend on the quadrupolar frequency and therefore the dependence of $C T$ on $v_{Q}$ occurs because of appearance satellite transitions during the QSP, which contribute to the value of $C T . T_{2, S T}$ of Pf1 is less than $T_{2, C T}: 8$ and 45 $\mathrm{ms}$, approximately ${ }^{32}$. Therefore, when the length of the QSP is too long, like in the first three points of Fig. 12c, the contribution of the satellite transitions decreases because of the influence of $T_{2, S T}$. The problem of relaxation in QSP experiments when $\tau_{p} \sim T_{2, S T}$ can be avoided, however, by acquiring more points between $\kappa \in(2: 4]$.

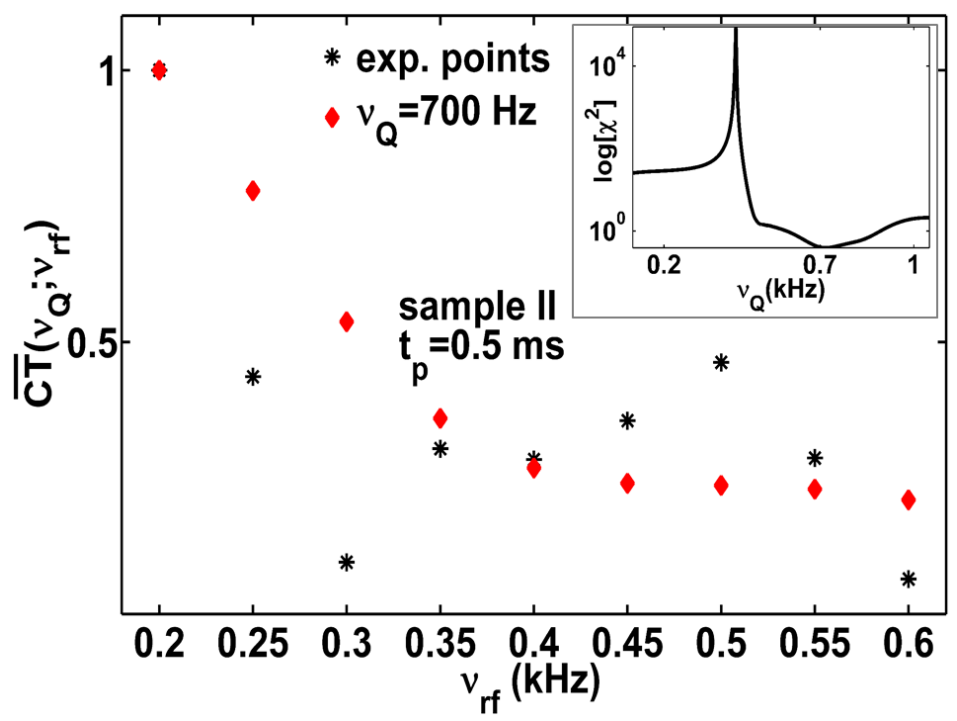

Fig. $13 \overline{C T}\left(v_{Q} ; v_{r f}\right)$ curves of sample II. The length of the pulses (except the $\pi$-pulse in the middle) is $0.5 \mathrm{~ms}$ and the total time of the QSP is $2.83 \mathrm{~ms}$. The rf-field of the $\pi$ - pulse is $600 \mathrm{~Hz}$.

We now investigate cartilage samples, which are known to display broad residual quadrupolar coupling distributions. Fig. 14 shows a $90^{\circ}$ spectrum of two prepared samples: sample IV in a quartz tube (a), sample $\mathrm{V}$ in a glass tube (b). In the first case the width of the background spectrum is about $2 \mathrm{kHz}$ and in the second case it is about $3 \mathrm{kHz}$. The difference in the widths of the spectra arises due to the contributions of the sodium signal from the glass. It can clearly be seen in the insert of Fig. 14b that the width of the background spectrum after the QSP sequence is approximately $8 \mathrm{kHz}$. As is shown below, despite of significant background noise, QSP is still able to extract quadrupolar couplings. 

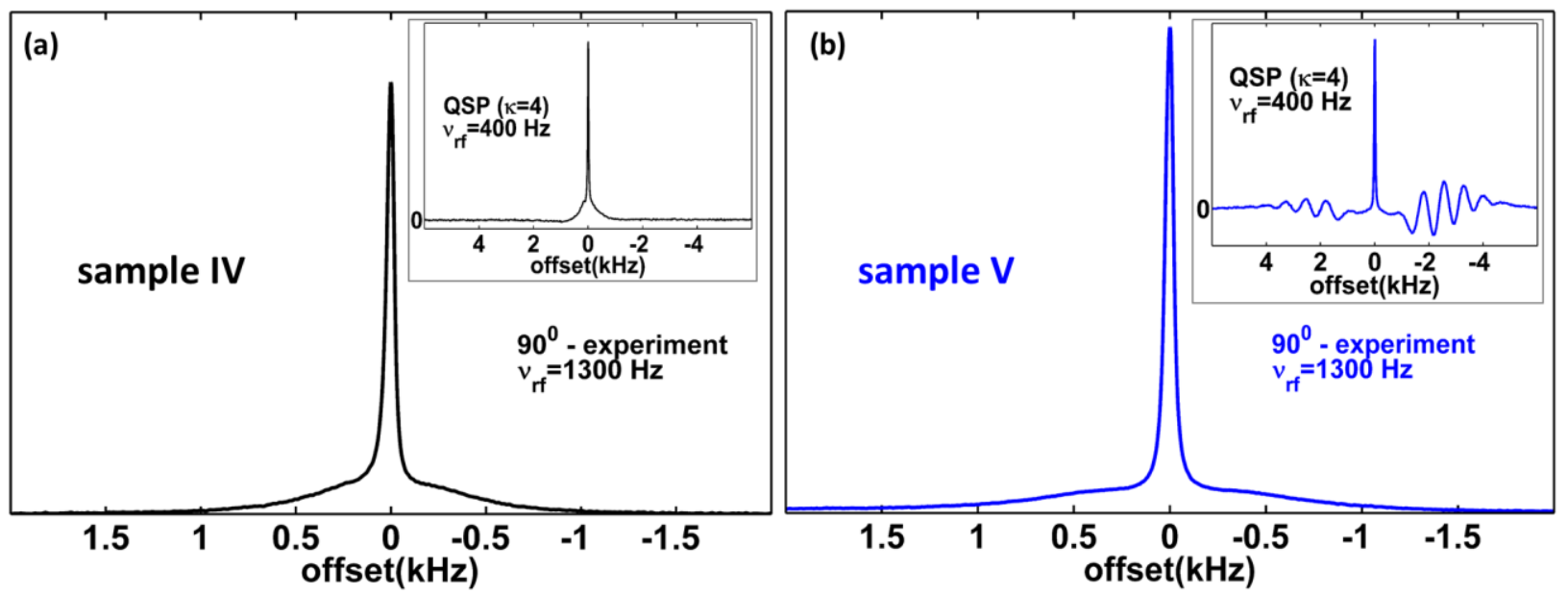

Fig. 14 The $90^{0}$ spectra of ${ }^{23} \mathrm{Na}$ in Cartilage in a quartz tube (a, sample IV) and in a glass tube (b, sample V). In both cases $v_{r f}$ is $1300 \mathrm{~Hz}$. Inserts: QSP spectrums of these samples under $n=4$ and $v_{r f}=400 \mathrm{~Hz}$.

In Fig. 15 we show 6 QSP curves for sample IV $(a, b, c)$ and V (d, e, f). The experimental points were compared to the simulations with a uniform orientational distribution (Eq. (4)). The obtained quadrupolar frequencies are shown in Table 3.
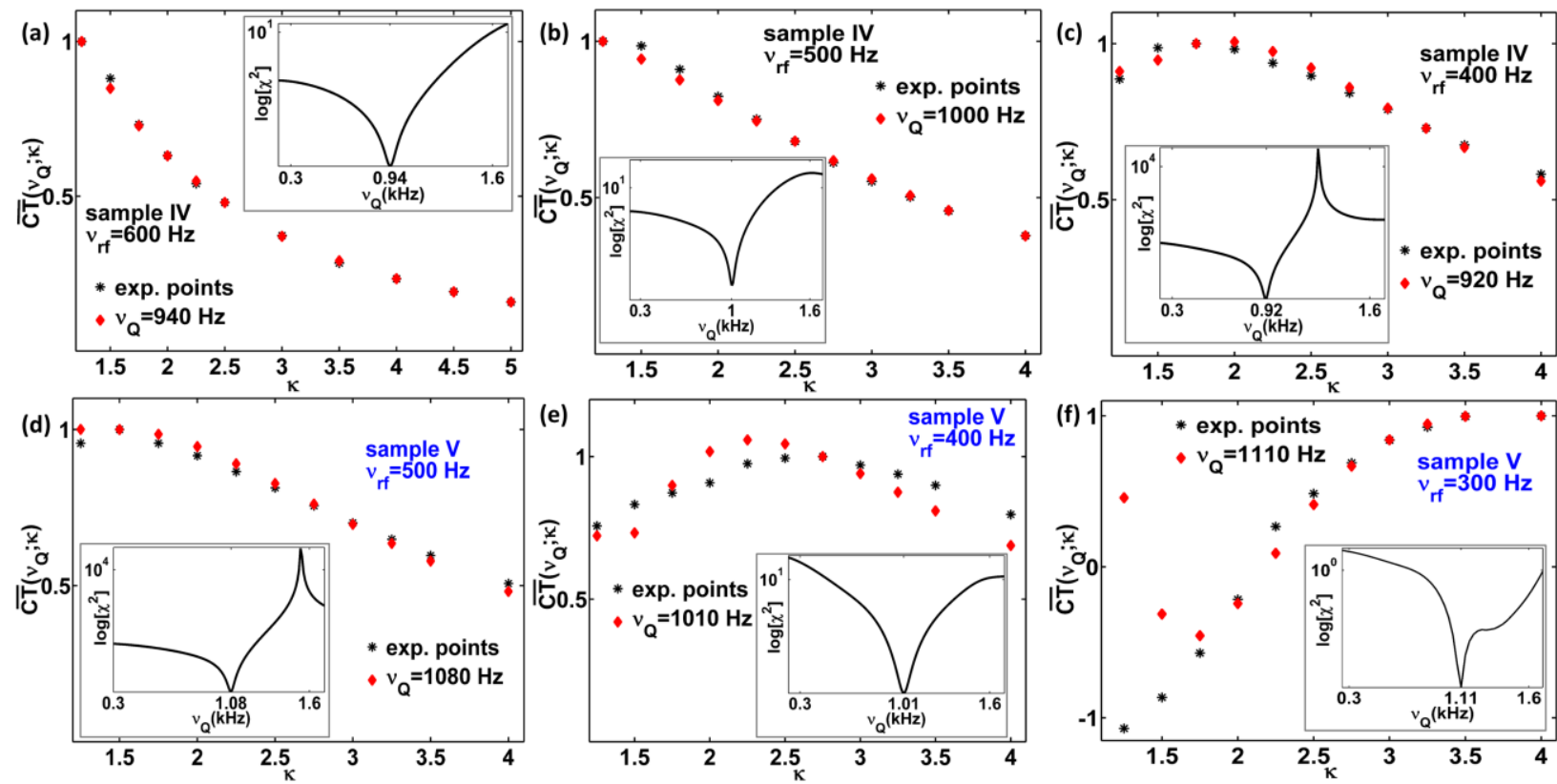

Fig. $15 \overline{C T}\left(v_{Q} ; \kappa\right)$ curves of sample IV (a, b, c) and sample V (d, e, f). (a) $v_{r f}=600 \mathrm{~Hz}$; (b) $v_{r f}=500 \mathrm{~Hz}$; (c) $v_{r f}=400 \mathrm{~Hz}$; (d) $v_{r f}=500 \mathrm{~Hz}$; (e) $v_{r f}=400 \mathrm{~Hz}$; (f) $v_{r f}=300 \mathrm{~Hz}$. The inserts show the logarithm of the residual from least squares fitting as a function of quadrupolar frequency. The number of the powder orientations for the simulations is 50 .

Table 3. The obtained quadrupolar frequencies with QSP - experiments under different $v_{r f}$ (Fig. 15).

\begin{tabular}{|c|c|c|c|c|}
\hline sample IV & $v_{r f}=600 \mathrm{~Hz}$ & $v_{r f}=500 \mathrm{~Hz}$ & $v_{r f}=400 \mathrm{~Hz}$ & average $(\mathrm{Hz})$ \\
\hline$v_{Q}(\mathrm{~Hz})$ & 940 & 1000 & 920 & 953 \\
\hline sample V & $v_{r f}=500 \mathrm{~Hz}$ & $v_{r f}=400 \mathrm{~Hz}$ & $v_{r f}=300 \mathrm{~Hz}$ & average \\
\hline$v_{Q}(\mathrm{~Hz})$ & 1080 & 1010 & 1110 & 1067 \\
\hline
\end{tabular}


The behaviors of the first experimental points in Fig. $15 \mathrm{f}$ differ from simulation, which is likely due to relaxation effects $\left(T_{2, S T}\right)$, which were described in the case of Pf1. The average quadrupolar frequencies of sample IV (quartz) and sample V (glass) are close to each other: $953 \mathrm{~Hz}$ and $1067 \mathrm{~Hz}$, respectively. The reason of the small divergence is due to signal of sodium in glass.

It is seen that averaging over a random distribution of principal axis frames produces acceptable results (Eq. (4)). Cartilage tissues often consist of compartments among which the ions exchange very slowly ${ }^{9}$ and the measured signal is described by Eq. (5). Because of the way the samples were prepared (three weeks storage of the cartilage in the refrigerator before experiments, cutting of the cartilage with a scalpel into strips and random packing into tubes), it is reasonable to assume such a description of the distribution of couplings. Therefore, these results suggest that the distribution of orientations can be characterized by this pulse sequence.

In Fig. 16a, we show the investigation of a sample of cartilage with additional added $\mathrm{NaCl}$ solution. We show the $90^{\circ}$ spectrum of sample IV with a streak of $\mathrm{NaCl}$ over a fluorinated oil solution (red spectrum) and the spectrum without $\mathrm{NaCl}$ (black spectrum). The ratio of the black peak to the red peak is about 1:4. Comparing the intensities of $C T(\kappa=1.25)$ of sample IV, sample IV $+\mathrm{NaCl}$ (not shown here) and Fig. 16a, we find that the ratio of ordered sodium in cartilage and free sodium in $\mathrm{NaCl}$ in that sample was 1:6.5. In Fig. 16b we show measured QSP curves of this sample at an rf-field of $600 \mathrm{~Hz}$. The extracted quadrupolar frequency with and without added $\mathrm{NaCl}$ solution are close to each other: $1050 \mathrm{~Hz}$ and 940 $\mathrm{Hz}$, respectively. The reasons of the small divergence are could be due to sodium diffusion in $\mathrm{NaCl}$ solution and inhomogeneity of the rf-field.
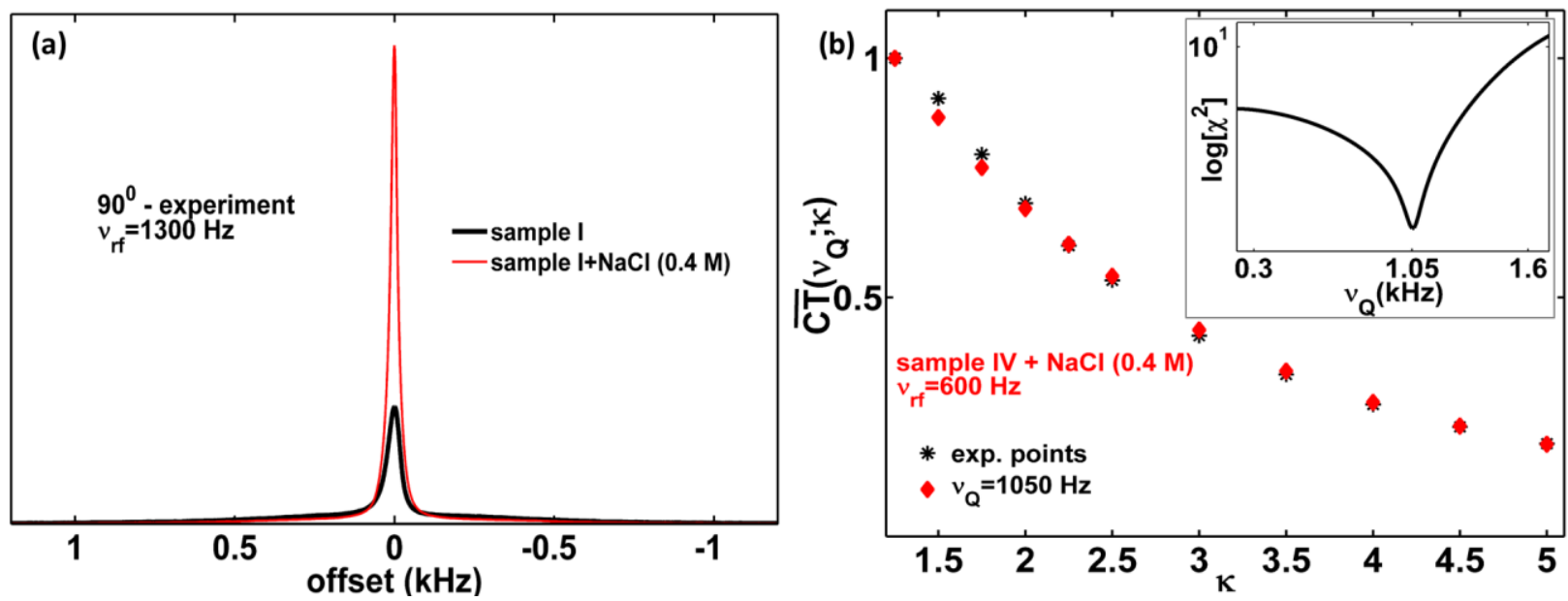

Fig. 16 (a) The $90^{0}$ spectra of ${ }^{23} \mathrm{Na}$ in Cartilage in a quartz tube (sample I) with added $\mathrm{NaCl}$ (red line) and comparison with a sample without $\mathrm{NaCl}$ (black line). (b) $\overline{C T}\left(v_{Q} ; \kappa\right)$ curve of sample I $+\mathrm{NaCl}$. $v_{r f}=600 \mathrm{~Hz}$.

In this section we showed QSP experiments with ${ }^{23} \mathrm{Na}$ in $\mathrm{NaCl}$ solution, Pf1 solutions and cartilage samples. The QSP has negligible influence on the isotropic spins. The quadrupolar frequencies of all Pf1 samples, which were obtained by QSP experiments are close to the frequencies with direct measurements. The quadrupolar frequencies of the all cartilage samples are close to each other. The experiments with cartilage samples in a glass tube and cartilage with $\mathrm{NaCl}$ showed that this sequence does not require special conditions for conducting the experiments. Also the quantitative explanation is provided about influence of the spin-spin relaxation on obtaining of the quadrupolar couplings. The relaxation effects 
have significant influences under $\tau_{p} \sim T_{2, S T}$ and they can be reduced using more short pulses $\kappa>2$ and taking more points in the area of $\kappa \in(2: 4]$.

\section{Conclusion}

Currently, all known methods for quantifying quadrupolar couplings of sodium in tissues require the use of relatively hard pulses and often extensive phase cycles. On an MRI scanner, or in ex situ applications, one often cannot use such ideal pulses. As a result, the sensitivity in double-quantum filtered experiments is relatively low and these sequences do not perform according to theory in these situations. Furthermore, broad background signals from more solid components could contaminate spectra and prevent accurate quantification. In this article, we have demonstrated a new method for the selective excitation of ordered sodium signals using weak pulses only. Under conditions where $v_{Q}=3 v_{r f}$ and $\kappa=2$ the intensity of the $C T$ after QSP can reach the same value as the intensity of the $C T$ after a $90^{\circ}$ pulse in the case of a single quadrupolar coupling frequency. For a random distribution of principal axis frames under conditions where $v_{Q}=1.25 v_{r f}$ and $\kappa=1.25$, the intensity of the $C T$ after QSP can achieve about $58 \%$ of the intensity of the $C T$ after $90^{\circ}$ pulse. Because of the structure of the sequence, the spatial inhomogeneity of the rf-field does not lead to the excitation of the isotropic spins and has a small influence on ordered spins (within 10\%). Furthermore, QSP could be used as a readout step in inversion recovery or as an excitation module in spin echo sequences, or simply for the selective excitation of ordered spins only. We have outlined here how the parameters of the sequence would be adjusted in order to find optimal conditions $\left(v_{r f} ; \kappa\right)$ for a specific range of quadrupolar frequencies. Since all this can be achieved with low power, these techniques could become useful under in vivo conditions, or in ex situ applications.

\section{Acknowledgments}

The Bruker Avance - 500 NMR Spectrometer was acquired through the support of the National Science Foundation under Award Number CHE 01162222. We acknowledge funding from the U.S. National Science Foundation, award No. CHE 1412064 for methodology development, and NIH R01AR060238 and NIH R01EB016045 for tissue-related work. We also thank Dr. Uzi Eliav from Tel Aviv University for pointing us to a number of helpful references.

1. Linzell, J. L. \& Peaker, M. Intracellular concentrations of sodium, potassium and chloride in the lactating mammary gland and their relation to the secretory mechanism. J. Physiol. 216, 683-700 (1971).

2. Madelin, G., Lee, J., Regatte, R. R. \& Jerschow, A. Sodium MRI : Methods and applications. Program 79, 14-47 (2014).

3. Rooney, W. D. \& Springer, J. A Comprehensive Approach to the Analysis and Interpretation of the Resonances of Spins 3 / 2 from-Living Systems. NMR Biomed. 4, 209-226 (1991).

4. Eliav, U. \& Navon, G. Sodium NMR/MRI for anisotropic systems. NMR Biomed. (2015).

5. Chung, C. \& Wimperis, S. Optimum Detection of Spin-3/2 Biexponential Relaxation Using Multiple-Quantum Filtration Techniques. J. Magn. Reson. 88, 440-447 (1990). 
6. Keinan-Adamsky, K. et al. $23 \mathrm{Na}$ and $2 \mathrm{H}$ magnetic resonance studies of osteoarthritic and osteoporotic articular cartilage. Magn. Reson. Med. 64, 653-661 (2010).

7. Kemp-Harper, R., Brown, S. P., Styles, P. \& Wimperis, S. In vivo NMR of sodium ions in ordered environments. J. Magn. Reson. B 105, 199-203 (1994).

8. Kemp-Harper, R. \& Wimperis, S. Detection of the Interaction of Sodium Ions with Ordered Structures in Biological Systems. Use of the Jeener-Broekaert Experiment. J. Magn. Reson. B 102, 326-331 (1993).

9. Eliav, U. \& Navon, G. Analysis of double-quantum-filtered NMR spectra of $23 \mathrm{Na}$ in biological tissues. J. Magn. Reson. B 103, 19-29 (1994).

10. Shinar, H., Knubovets, T., Eliav, U. \& Navon, G. Sodium interaction with ordered structures in mammalian red blood cells detected by Na-23 double quantum NMR. Biophys. J. 64, 1273-1279 (1993).

11. Eliav, U. \& Navon, G. Quadrupole-Echo Techniques in Multiple-Quantum-Filtered NMR Spectroscopy of Heterogeneous Systems. J. Magn. Reson. A 115, 241-253 (1995).

12. Brown, S. P. \& Wimperis, S. Extraction of Homogeneous 23 Na NMR Linewidths from TwoDimensional Jeener - Broekaert Spectra*. J. Magn. Reson. 109, 291-300 (1995).

13. Pekar, J. \& Leigh, J. S. Detection of biexponential relaxation in sodium-23 facilitated by doublequantum filtering. J. Magn. Reson. 69, 582-584 (1986).

14. Brown, S. P. \& Wimperis, S. NMR measurement of spin-3/2 transverse relaxation in an inhomogeneous B1 field. Chem. Phys. Lett. 224, 508-516 (1994).

15. Hughes, C. E., Kemp-Harper, R., Styles, P. \& Wimperis, S. NMR Spectroscopy and Imaging of Sodium in Ordered Environments. The Return of the Central Transition. J. Magn. Reson. B 111, 189-193 (1996).

16. Lee, J., Regatte, R. R. \& Jerschow, A. Optimal control NMR differentiation between fast and slow sodium. Chem. Phys. Lett. 494, 331-336 (2010).

17. Tsang, A., Stobbe, R. W. \& Beaulieu, C. Triple-quantum-filtered sodium imaging of the human brain at 4.7 T. Magn. Reson. Med. 67, 1633-1643 (2012).

18. Eykyn, T. R. et al. Multiple quantum filtered 23Na NMR in the Langendorff perfused mouse heart: Ratio of triple/double quantum filtered signals correlates with [Na]i. J. Mol. Cell. Cardiol. 86, 95-101 (2015).

19. Choy, J., Ling, W. \& Jerschow, A. Selective detection of ordered sodium signals via the central transition. J. Magn. Reson. 180, 105-109 (2006).

20. Eliav, U., Keinan-Adamsky, K. \& Navon, G. A new method for suppressing the central transition in $\mathrm{I}=3 / 2 \mathrm{NMR}$ spectra with a demonstration for $23 \mathrm{Na}$ in bovine articular cartilage. J. Magn. Reson. 165, 276-281 (2003).

21. Lee, J., Regatte, R. R. \& Jerschow, A. Selective detection of ordered sodium signals by a jumpand-return pulse sequence. J. Magn. Reson. 200, 126-129 (2009).

22. Reddy, R., Bolinger, L., Shinnar, M., Noyszewski, E. \& Leigh, J. S. Detection of residual quadrupolar interaction in human skeletal muscle and brain in vivo via multiple quantum filtered sodium NMR spectra. Magn. Reson. Med. 33, 134-139 (1995).

23. Pound, R. V. Nuclear Electric Quadrupole Interactions in Crystals. Phys. Rev. 79, 685-702 (1950).

24. Slichter, C. P. in Principles of Magnetic Resonance 485-502 (1996).

25. Halle, B. Theory of intramolecular spin relaxation by translational diffusion in locally ordered 
fluids. Mol. Phys. 60, 319-370 (1987).

26. Kemp-Harper, R., Wickstead, B. \& Wimperis, S. Sodium ions in ordered environments in biological systems: analysis of 23Na NMR spectra. J. Magn. Reson. 140, 351-362 (1999).

27. Conroy, H. Molecular Schrödinger Equation. VIII. A New Method for the Evaluation of Multidimensional Integrals. J. Chem. Phys. 47, 5307-5318 (1967).

28. Kemp-Harper, R., Brown, S. P., Hughes, C. E. \& Wimperis, S. 23Na NMR methods for selective observation of sodium ions in ordered environments. Prog. Nucl. Magn. Reson. Spectrosc. 30, 157-181 (1997).

29. Vega, S. \& Naor, Y. Triple quantum NMR on spin systems with $\mathrm{I}=3 / 2$ in solids. J. Chem. Phys. 75, 75-86 (1981).

30. Shekar, S. C., Tang, J. A. \& Jerschow, A. Dynamics of I=3/2 Nuclei in Isotropic Slow Motion, Anisotropic and Partially Ordered Phases. Concepts Magn. Reson. Part A 36A, 362-387 (2010).

31. Sobieski, D. N., Krueger, N. R., Vyas, S. \& Augustine, M. P. Nuclear spin relaxation of sodium cations in bacteriophage Pf1 solutions. J. Chem. Phys. 125, 244509 (2006).

32. Lee, J., Regatte, R. R. \& Jerschow, A. Optimal excitation of N 23 a nuclear spins in the presence of residual quadrupolar coupling and quadrupolar relaxation. J. Chem. Phys. 131, 1-8 (2009). 
Quadrupole Sensitive Pulse for signal filtering
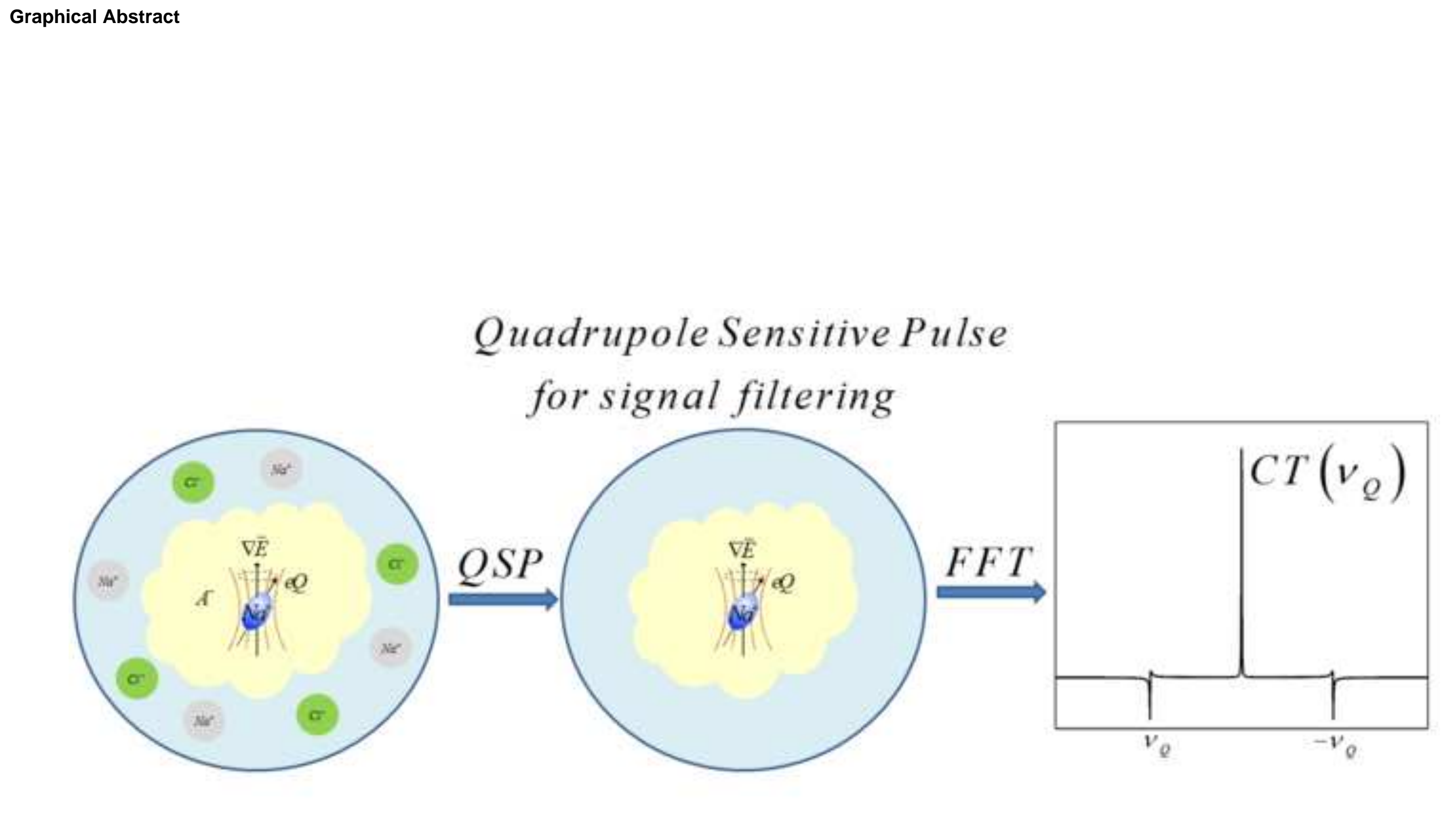

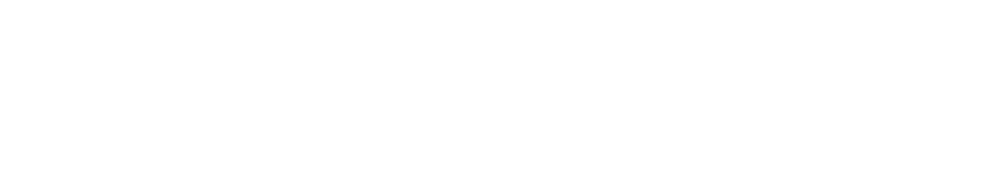

-

(a)

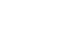

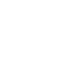

.

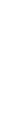

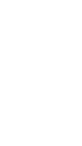

